\title{
Amplitude-phase description of stochastic neural oscillators across the Hopf bifurcation
}

\author{
Arthur S. Powanwe (1)* and André Longtin $~^{*}{ }^{\dagger}$ \\ Physics Department and Centre for Neural Dynamics, University of Ottawa, 150 Louis Pasteur, Ottawa, Ontario, Canada K1N 6 N5
}

(Received 8 December 2020; accepted 7 June 2021; published 9 July 2021)

\begin{abstract}
We derive a unified amplitude-phase decomposition for both noisy limit cycles and quasicycles; in the latter case, the oscillatory motion has no deterministic counterpart. We extend a previous amplitude-phase decomposition approach using the stochastic averaging method (SAM) for quasicycles by taking into account nonlinear terms up to order 3 . We further take into account the case of coupled networks where each isolated network can be in a quasi- or noisy limit-cycle regime. The method is illustrated on two models which exhibit a deterministic supercritical Hopf bifurcation: the Stochastic Wilson-Cowan model of neural rhythms, and the Stochastic Stuart-Landau model in physics. At the level of a single oscillatory module, the amplitude process of each of these models decouples from the phase process to the lowest order, allowing a Fokker-Planck estimate of the amplitude probability density. The peak of this density captures well the transition between the two regimes. The model describes accurately the effect of Gaussian white noise as well as of correlated noise. Bursting epochs in the limit-cycle regime are in fact favored by noise with shorter correlation time or stronger intensity. Quasicycle and noisy limit-cycle dynamics are associated with, respectively, Rayleigh-type and Gaussian-like amplitude densities. This provides an additional tool to distinguish quasicycle from limit-cycle origins of bursty rhythms. The case of multiple oscillatory modules with excitatory all-to-all delayed coupling results in a system of stochastic coupled amplitude-phase equations that keeps all the biophysical parameters of the initial networks and again works across the Hopf bifurcation. The theory is illustrated for small heterogeneous networks of oscillatory modules. Numerical simulations of the amplitude-phase dynamics obtained through the SAM are in good agreement with those of the original oscillatory networks. In the deterministic and nearly identical oscillators limits, the stochastic Stuart-Landau model leads to the stochastic Kuramoto model of interacting phases. The approach can be tailored to networks with different frequency, topology, and stochastic inputs, thus providing a general and flexible framework to analyze noisy oscillations continuously across the underlying deterministic bifurcation.
\end{abstract}

DOI: 10.1103/PhysRevResearch.3.033040

\section{INTRODUCTION}

Limit cycle oscillations have been studied in many areas ranging from biology [1-3], ecology [4,5], and laser physics [6]. Biological limit cycles in particular have been the subject of numerous papers over the past decades in the context of biochemical oscillations [7-11], circadian rhythms [12,13], genetic oscillations [14-18], cardiac rhythms [19,20], calcium oscillations [21,22], epidemic and ecological oscillations [23-26], neural rhythms [27-31], and hair cell motion [32,33]. These rhythms often display strong fluctuations, or are seen during brief, randomly recurring epochs. In certain systems such as circadian rhythms, the oscillations are known to be an essential part of the function. In neural systems, slow (delta,alpha) [34,35] and fast (beta, gamma) [28,36,37] neural

\footnotetext{
*apowa074@uottawa.ca

†alongtin@uottawa.ca
}

Published by the American Physical Society under the terms of the Creative Commons Attribution 4.0 International license. Further distribution of this work must maintain attribution to the author(s) and the published article's title, journal citation, and DOI. rhythms are believed to support cognitive processes such as perception [38], attention [39], cognition [40], working memory $[41,42]$, or communication between brain areas $[43,44]$. Abnormal or dysfunctional rhythms have been linked to neurological disorders like epilepsy, Alzheimer disease (AD), and Parkingson's disease (PD) [45-47] just to mention a few.

Amplitude-phase descriptions of limit-cycle oscillations have been investigated using a variety of techniques [48-54] following early studies of noise-induced and noise-perturbed oscillations [55-57]. The focus has been mainly on the phase, as studies often assume weak noise which leads to small amplitude variations. Thus the amplitude-phase description is often reduced to a simple phase description of the noisy limit-cycle oscillations [58]. Recently, descriptions in terms of the slow amplitude fluctuations and phase, referred henceforth as amplitude-phase, have been applied to the quasicycle regime and their random bursts $[59,60]$. Bursts correspond to epochs of higher amplitude values. In this regime, a simple phase description of the oscillation is unable to capture the bursting features, and thus taking the amplitude into account is necessary. Our goal is to provide a framework for computing amplitude-phase equations across the Hopf bifurcation for single oscillatory modules, driven by white or colored noise processes, and to generalize this approach to networks of 
delay-coupled modules. This will pave the way to understand communication with bursts across brain areas.

There is increasing evidence that fast rhythms in certain neural systems are induced rather than perturbed by noise [60-63]. Similar observations have been made for predator-prey ecological systems, whose overall cyclical activity has been suggested to require demographic noise [26]. The vicinity of the transition between noisy limit cycles and noise-induced limit cycles known as "quasicycles" is thus worthy of attention as systems may drift over this boundary.

The modeling of stochastic oscillations thus involves the distinction between noisy limit cycles and quasicycles, depending on whether one suspects the deterministic dynamics to lie below or beyond a bifurcation from a fixed point to a limit cycle. Noisy limit cycles are self-sustained oscillations which exist independently of their noisy environment, i.e. are a manifestation of operation beyond the bifurcation. They are modelled as single or coupled oscillators. Quasicycles on the other hand are induced and sustained by their stochastic surroundings, in the absence of which trajectories decay to a fixed point. Models for quasicycles assume stable complex eigenvalues around this fixed point [59].

A unified framework which smoothly meshes together these two classes of stochastic models at the level of single as well as coupled networks is of interest, in particular when the focus is a decomposition into their time-dependent amplitude and phase. Previous studies have described the normal form dynamics of the Hopf bifurcation with additive and multiplicative noise $[57,64,65]$, including the multi-scale behavior known to occur in the vicinity of the bifurcation [66]. The seminal study in [64] does perform a transformation of a system of arbitrary dimension near a Hopf bifurcation into a set of dynamical equations for the complex mode amplitudes; further work would then be required to convert such a system into an amplitude-phase description as is our end goal here. It also does not produce a slower amplitude dynamics which is of interest to eventually characterize bursting. Our work below starts instead with additive colored noise and different noise on the components, and derives slow amplitude dynamics. Further it goes beyond considering a single oscillatory system; although each system is in $2 \mathrm{D}$, we develop the theory for several delay-coupled 2D systems. In contrast, in the context of neural rhythms, current models that describe quasicycles and their coupled counterparts are based simply on linear equations driven by noise and are confined below the Hopf bifurcation [59,60,67-69]. Thus, we are not aware of any method that directly yields an amplitude-phase representation of coupled neural networks that is accurate across the Hopf bifurcation, let alone one that can handle colored noise and delay coupling.

Our study derives such a decomposition that bridges the gap across this boundary, i.e., across the Hopf bifurcation for both single and coupled networks. The slow amplitude that emerges from our analysis quantifies the temporal evolution of the cycle to cycle amplitude of the rhythm, while the phase contains information about its regularity. This is done by extending a previous amplitude-phase decomposition of quasicycles oscillation to noisy limit cycles by considering nonlinear terms up to order three in the fast dynamics. This involves lengthier calculations using the stochastic av- eraging method (SAM) [70-72]. While the Taylor expansion of sigmoidal nonlinearities like those of the neural response functions converge slowly, our immediate goal is only to obtain the next order corrections to our previous nonlinear amplitude-phase equations derived from linear dynamics. Our method can accurately describe noisy oscillations over a range of frequencies. It can also accommodate different types of noise across a range of intensities. In fact the general theory in our work also considers quasicycle and limit-cycle dynamics driven by Ornstein-Uhlenbeck (OU) noise.

We develop our theory using two models of rhythm generation, namely, the stochastic Wilson-Cowan model (SWC) of neural rhythms [31,73], and the stochastic Stuart-Landau model (SSL) used to describe oscillations in various areas including physics and neuroscience. Each two-dimensional model system describes the interaction of a first variable ( $E$ or $x$ ) with a second variable ( $I$ or $y$ ). Multiple such systems have been used to study the emergence and coupling of rhythms across brain areas [74]. The results of such analyses have even been shown to account for features of real data [75-77]. Both models exhibit a supercritical Hopf bifurcation; in fact the Stuart-Landau model in polar coordinates is the normal form for this bifurcation [78]. Thus our illustrative examples are taken from the field of neural rhythms and physics, but are more generally applicable.

We further generalize our results for a two-population $E-I$ network model to super-networks whose unit building blocks are such $E-I$ modules, each of which can oscillate on its own in either the quasi or limit-cycle regime. Our amplitude-phase model goes beyond purely phase-coupled oscillator models to describe both the quasicycle and noisy limit-cycle dynamics with a higher level of biophysical realism. It also allows moderate noise and coupling strength. The model is adaptable to different frequency bands, and can be simplified to obtain a representation in terms of coupled Kuramoto oscillators.

We focus our work on the amplitude dynamics, showing good agreement with the original nonlinear model in both the quasi- and limit-cycle regimes. Coupled rhythm-generating networks have been considered in the context of the activity arising from the brain connectome [76], as well as genetic and biochemical networks [7]. The modeling of such networks has mostly been limit-cycle-based in the deterministic or weak noise limit, and assumed weakly coupled and nearly identical oscillators. In such limits, the influence of the amplitudes of the oscillators is usually neglected and the only important variables are the phases of the oscillatory units. Such interacting phase models do not describe coupled quasicycles whose behavior is strongly amplitude-dependent. We note however a recent study of coupled quasicycles that involves amplitudephase coupling [68]. In contrast, our study of supernetworks in the last part of our paper involves quasicycle dynamics and noisy limit cycles.

We first present the nonlinear version of the stochastic Wilson-Cowan model, with its third order approximation and derive the amplitude-phase approximation that agrees well with the numerics of the original model across the Hopf bifurcation. We then perform a similar calculation for the stochastic Stuart-Landau model. Finally, supernetworks of such models are considered. Our theory is developed assuming that these systems are driven by OU noise. For simplicity, most of our 
simulations are compared with the theory for the white noise case; the exceptions are Figures 10 and 11 where the effect of the noise correlation time on amplitudes densities is highlighted.

\section{STOCHASTIC WILSON-COWAN MODEL}

We consider a stochastic version [31] of the seminal Wilson-Cowan model [73] for oscillation generation. The two-dimensional model with additive noise mimics a population of excitatory $(E)$ and of inhibitory $(I)$ cells. The mean synaptic coupling from $E$ cells to themselves, and from $E$ cells to $I$ cells, are given respectively by $W_{E E}$ and $W_{I E}$. Similarly, the mean synaptic coupling from $I$ cells to themselves and from $I$ cells to $E$ cells are $W_{I I}$ and $W_{E I}$. The $E$ cell population receives a constant external stimulus $h_{E}$, while the $I$ cell population is driven by the constant external current $h_{I}$. Additive noise is assumed to arise mainly from random synaptic events. Defining the fraction of excitatory cells that are firing at a given time by $E(t)$, and of inhibitory cells $I(t)$, yields the stochastic WC model (SWC):

$$
\begin{gathered}
\frac{d E(t)}{d t}=-\alpha_{E} E(t)+(1-E(t)) \beta_{E} f\left(s_{E}(t)\right)+\eta_{E}(t), \\
\frac{d I(t)}{d t}=-\alpha_{I} I(t)+(1-I(t)) \beta_{I} f\left(s_{I}(t)\right)+\eta_{I}(t) .
\end{gathered}
$$

The sigmoid function is $f(x)=\left(1+e^{-x}\right)^{-1}$ and the total excitatory $s_{E}(t)$ and inhibitory $s_{I}(t)$ synaptic inputs to a neuron are given respectively by

$$
\begin{gathered}
s_{E}(t)=W_{E E} E(t)-W_{E I} I(t)+h_{E}, \\
s_{I}(t)=W_{I E} E(t)-W_{I I} I(t)+h_{I} .
\end{gathered}
$$

We choose $\eta_{E, I}(t)$ as two independent Ornstein-Uhlenbeck (OU) [79] processes given by

$$
\begin{aligned}
& \frac{d \eta_{E}(t)}{d t}=-\frac{\eta_{E}(t)}{\tau_{E}}+\sqrt{\frac{2 \sigma_{E}^{2}}{\tau_{E}}} \xi_{E}(t), \\
& \frac{d \eta_{I}(t)}{d t}=-\frac{\eta_{I}(t)}{\tau_{I}}+\sqrt{\frac{2 \sigma_{I}^{2}}{\tau_{I}}} \xi_{I}(t),
\end{aligned}
$$

where $\xi_{E, I}$ are two independent Gaussian white noises with the following properties:

$$
\begin{aligned}
\left\langle\xi_{E, I}(t)\right\rangle & =0, \quad\left\langle\xi_{E}(t) \xi_{I}(t)\right\rangle=0, \\
\left\langle\xi_{E}\left(t^{\prime}\right) \xi_{E}(t)\right\rangle & =\left\langle\xi_{I}\left(t^{\prime}\right) \xi_{I}(t)\right\rangle=\delta\left(t-t^{\prime}\right) .
\end{aligned}
$$

The variances of these OU processes are $\sigma_{E, I}^{2}$, their autocorrelations are given by

$$
\begin{aligned}
\left\langle\eta_{E}(t) \eta_{E}(s)\right\rangle & =\sigma_{E}^{2} \exp \left(-|t-s| / \tau_{E}\right), \\
\left\langle\eta_{I}(t) \eta_{I}(s)\right\rangle & =\sigma_{I}^{2} \exp \left(-|t-s| / \tau_{I}\right),
\end{aligned}
$$

and their intensities (integrals of the autocorrelation functions) are $Q_{E, I}=\tau_{E, I} \sigma_{E, I}^{2}$. Further in our work, we will investigate the effect of the noise correlation time on the dynamics, while maintaining the noise intensity fixed. In other words, we will fix $Q_{E, I}$, and vary $\tau_{E, I}$ while changing $\sigma_{E, I}^{2}$. However, for the stochastic Wilson-Cowan model in the first part of our paper, we will consider stochastic forcing by Gaussian white noises.
The noise-free $\left(\sigma_{E}=\sigma_{I}=0\right)$ dynamics has a fixed point $\left(E_{0}, I_{0}\right)$ (for the parameter values chosen in our work) which can be a stable or an unstable focus. If the fixed point is a stable focus, deviations from the fixed point converge to zero. The noise slowly erases the memory of the initial conditions, and induces deviations from the fixed point that relax in a noisy oscillatory manner $[59,60]$. Adding noise to this regime leads to noise-induced oscillations or quasicycles. If the fixed point is an unstable focus, the system undergoes a Hopf bifurcation to a stable limit cycle. The noise then yields a stochastic limit cycle $[80,81]$ by perturbing the amplitude, frequency and phase of the deterministic oscillation.

\section{A. Dynamics of the fluctuations}

We wish to characterize the fast and slow features of the deviations from the fixed point with a unique dynamical description no matter if their corresponding dynamics is a quasicycle or a noisy limit cycle. We look for solutions of Eqs. (1) and (2) in the form

$$
E(t)=E_{0}+V_{E}(t), \quad I(t)=I_{0}+V_{I}(t),
$$

where the fixed points $E_{0}$ and $I_{0}$ solve the deterministic equations:

$$
\begin{aligned}
-\alpha_{E} E_{0}+\left(1-E_{0}\right) \beta_{E} f\left(s_{E_{0}}\right) & =0, \\
-\alpha_{I} I_{0}+\left(1-I_{0}\right) \beta_{I} f\left(s_{I_{0}}\right) & =0,
\end{aligned}
$$

with the definitions

$$
\begin{gathered}
s_{E_{0}}=W_{E E} E_{0}-W_{E I} I_{0}+h_{E}, \\
s_{I_{0}}=W_{I E} E_{0}-W_{I I} I_{0}+h_{I} .
\end{gathered}
$$

Note that we have restricted our analysis to the parameter regime most relevant to brain rhythms, namely, the one where the system has only one deterministic fixed point. We inserted Eqs. (9) into Eqs. (1) and (2) and used the following Taylor expansion of the sigmoid function:

$$
\begin{aligned}
f\left(s_{E}(t)\right) & =f\left(s_{E_{0}}+\delta s_{E}(t)\right) \\
& =f\left(s_{E_{0}}\right)+\delta s_{E}(t) f^{\prime}\left(s_{E_{0}}\right)+\frac{1}{2}\left(\delta s_{E}(t)\right)^{2} f^{\prime \prime}\left(s_{E_{0}}\right), \\
f\left(s_{I}(t)\right) & =f\left(s_{I_{0}}+\delta s_{I}(t)\right) \\
& =f\left(s_{I_{0}}\right)+\delta s_{I}(t) f^{\prime}\left(s_{I_{0}}\right)+\frac{1}{2}\left(\delta s_{I}(t)\right)^{2} f^{\prime \prime}\left(s_{I_{0}}\right),
\end{aligned}
$$

where

$$
\begin{gathered}
\delta s_{E}(t)=W_{E E} V_{E}(t)-W_{E I} V_{I}(t), \\
\delta s_{I}(t)=W_{I E} V_{E}(t)-W_{I I} V_{I}(t) .
\end{gathered}
$$

We note that the Taylor expansion made above in Eqs. (11) is accurate for small deviations in Eqs. (12). If these quantities become too large, this approximation becomes inaccurate which may lead to incorrect results.

The fluctuations $V_{E}(t)$ and $V_{I}(t)$ are considered as local field potentials (LFP) in the context of brain dynamics $[59,60]$. These LFPs are dimensionless in the Wilson-Cowan modeling framework since they are simply deviations from the steady state fractions $E_{0}$ and $I_{0}$; they are nevertheless interpreted as 
potentials [61]. They are approximately governed by

$$
\begin{aligned}
\frac{d V_{E}(t)}{d t}= & A_{E E} V_{E}(t)+A_{E I} V_{I}(t)+L_{E E} V_{E}^{2}(t)+L_{E I} V_{E}(t) V_{I}(t) \\
& +M_{E I} V_{I}^{2}(t)+B_{1 E} V_{E}^{3}(t)+B_{2 E} V_{E} V_{I}^{2}(t) \\
& +B_{3 E} V_{I} V_{E}^{2}(t)+B_{4 E} V_{I}^{3}(t)+\eta_{E}(t) \\
\frac{d V_{I}(t)}{d t}= & A_{I E} V_{E}(t)+A_{I I} V_{I}(t)+L_{I I} V_{I}^{2}(t)+L_{I E} V_{E}(t) V_{I}(t) \\
& +M_{I E} V_{E}^{2}(t)+B_{1 I} V_{I}^{3}(t)+B_{2 I} V_{E} V_{I}^{2}(t) \\
& +B_{3 I} V_{I} V_{E}^{2}(t)+B_{4 I} V_{E}^{3}(t)+\eta_{I}(t)
\end{aligned}
$$

with the following parameter definitions:

$$
\begin{aligned}
A_{E E} & =-\left[\alpha_{E}+\beta_{E} f\left(s_{E_{0}}\right)-\left(1-E_{0}\right) \beta_{E} f^{\prime}\left(s_{E_{0}}\right) W_{E E}\right], \\
A_{I I} & =-\left[\alpha_{I}+\beta_{I} f\left(s_{I_{0}}\right)+\left(1-I_{0}\right) \beta_{I} f^{\prime}\left(s_{I_{0}}\right) W_{I I}\right], \\
A_{E I} & =-\left(1-E_{0}\right) \beta_{E} f^{\prime}\left(s_{E_{0}}\right) W_{E I}, \\
A_{I E} & =\left(1-I_{0}\right) \beta_{I} f^{\prime}\left(s_{I_{0}}\right) W_{I E}, \\
L_{E E} & =-\beta_{E} f^{\prime}\left(s_{E_{0}}\right) W_{E E}, \quad L_{E I}=\beta_{E} f^{\prime}\left(s_{E_{0}}\right) W_{E I}, \\
L_{I E} & =-\beta_{I} f^{\prime}\left(s_{I_{0}}\right) W_{I E}, \quad L_{I I}=\beta_{I} f^{\prime}\left(s_{I_{0}}\right) W_{I I}, \\
M_{E I} & =0, \quad M_{I E}=0, \quad B_{4 E}=0, \quad B_{4 I}=0 ; \\
B_{1 E} & =-\frac{1}{2} \beta_{E} f^{\prime \prime}\left(s_{E_{0}}\right) W_{E E}^{2}, \quad B_{2 E}=-\frac{1}{2} \beta_{E} f^{\prime \prime}\left(s_{E_{0}}\right) W_{E I}^{2} \\
B_{3 E} & =\beta_{E} f^{\prime \prime}\left(s_{E_{0}}\right) W_{E E} W_{E I}, \quad B_{1 I}=-\frac{1}{2} \beta_{I} f^{\prime \prime}\left(s_{I_{0}}\right) W_{I I}^{2} \\
B_{2 I} & =\beta_{I} f^{\prime \prime}\left(s_{I_{0}}\right) W_{I E} W_{I I}, \quad B_{3 I}=-\frac{1}{2} \beta_{I} f^{\prime \prime}\left(s_{I_{0}}\right) W_{I E}^{2} .
\end{aligned}
$$

Note that to obtain Eqs. (13) and (14), we have used the fixed point condition Eq. (10). The Taylor expansion for this stochastic WC model leads to the four terms $M_{E I}=M_{I E}=$ $B_{4 E}=B_{4 I}=0$. The values of these parameters is a consequence of a specific choice of some terms which leads to a better convergence to the former Wilson-Cowan dynamics as we show in Appendix, Fig. 14. They could be different from zero in other models. For our analytic work, we will use Eqs. (13) and (14) instead of Eqs. (1) and (2). Equations (13) and (14) are the approximate fluctuation dynamics to order $\mathrm{O}(3)$ of the stochastic Wilson-Cowan model, denoted henceforth as SWC3 (see further below in Fig. 3 for time series from this model). We focus on this system and investigate its ability to generate oscillations and amplitude-phase dynamics across the Hopf boundary in the presence of noise.

\section{Nonlinear fluctuation dynamics: Numerical analysis}

We first simulate Eqs. (13) and (14) without stochastic inputs ( $\sigma_{E}=0$ and $\sigma_{I}=0$ ). Then we use Eqs. (9) to compute the synchronization level of any existing long-life oscillations of the excitatory activity $E(t)$. The synchronization level was defined as the mean of the amplitudes between each trough and the successive peak, computed using a very long simulation time. It can also be seen as the mean of the cycleto-cycle amplitudes of the oscillatory process after transients have decayed. We plotted this synchronization level in the $\left(\boldsymbol{W}_{\boldsymbol{E} E}, \boldsymbol{W}_{\boldsymbol{I I}}\right)$ parameter space in Fig. 1(a), which reveals the emergence of oscillations after a Hopf bifurcation. The level of synchronization increases smoothly for parameters deeper into the limit-cycle regime. Accordingly, the mean amplitude of the limit cycle increases with the real part of the complex conjugate eigenvalues at that fixed point.

We now take stochastic inputs into account. We first consider only white noises $\eta_{E, I}(t)=\sigma_{E, I} \xi_{E, I}(t)$ and define the total noise strength as

$$
\sigma=\sqrt{\sigma_{E}^{2}+\sigma_{I}^{2}} .
$$

For weak noise, quasicycle oscillations emerge [Fig. 1(b)]. In the limit-cycle regime, the oscillation amplitude is larger at higher noise [Fig. 1(c)] compared to the lower noise [Fig. 1(b)] and deterministic [Fig. 1(a)] cases. At the higher noise strength in Fig 1(c), the oscillations are in fact more prominent in both the quasicycle and limit-cycle regimes.

The oscillation frequency for weak noise strength is also shown in Fig. 1(d), where it is apparent that it varies smoothly across the Hopf boundary. The frequency content of the quasicycle oscillations can be seen in the spectrogram [Fig. 1(e)]. Oscillations are quite irregular, with epochs of high and low amplitude values. The epochs of high amplitude values are "bursts"; the mean frequency of such bursts is also a random variable. In contrast, in the limit-cycle regime [black dot in Fig. 1(b)] oscillations are more regular, with small fluctuations in amplitude and peak frequency as seen in the corresponding spectrogram [Fig. 1(f)].

\section{Linear analysis}

Recent studies showed that noise-induced oscillations could be described by linear equations sustained by noise $[59,60]$. More interestingly, it was found that the amplitude ratio and the phase difference between inhibitory and excitatory fluctuations were constant in the deterministic limit $[59,60]$. In the most general case considered here, the dynamics of excitatory and inhibitory fluctuations are described by nonlinear equations which are sustained by noise when the real part of the eigenvalues is negative and self-sustained when it is positive. We therefore checked whether these properties of the $E$ and $I$ amplitudes and phases are conserved in the regime of self-sustained oscillations. For that, we perform the linear analysis of Eqs. (13) and (14). The associated noise-free linear system of Eqs. (13) and (14) can be written in the following matrix form:

$$
\frac{d V^{0}(t)}{d t}=A V^{0}(t)
$$

where

$$
V^{0}(t)=\left[\begin{array}{c}
V_{E}^{0}(t) \\
V_{I}^{0}(t)
\end{array}\right] \text { and } A=\left[\begin{array}{cc}
A_{E E} & A_{E I} \\
A_{I E} & A_{I I}
\end{array}\right]
$$

We look for a trial solution in the form:

$$
\left[\begin{array}{l}
V_{E}^{0}(t) \\
V_{I}^{0}(t)
\end{array}\right]=\left[\begin{array}{c}
\tilde{B_{E}} \\
\tilde{B_{I}}
\end{array}\right] e^{\lambda t},
$$

where $\tilde{B_{E}}=B_{E} e^{j \theta_{E}}$ and $\tilde{B_{I}}=B_{I} e^{j \theta_{I}}$. The eigenvalue $\lambda$ of the associated matrix $A$ is found by substituting the trial solution into the linear system, yielding

$$
\frac{\tilde{B_{I}}}{\tilde{B_{E}}}=\frac{A_{E E}-\lambda}{-A_{E I}}=-\frac{A_{I E}}{A_{I I}-\lambda} .
$$



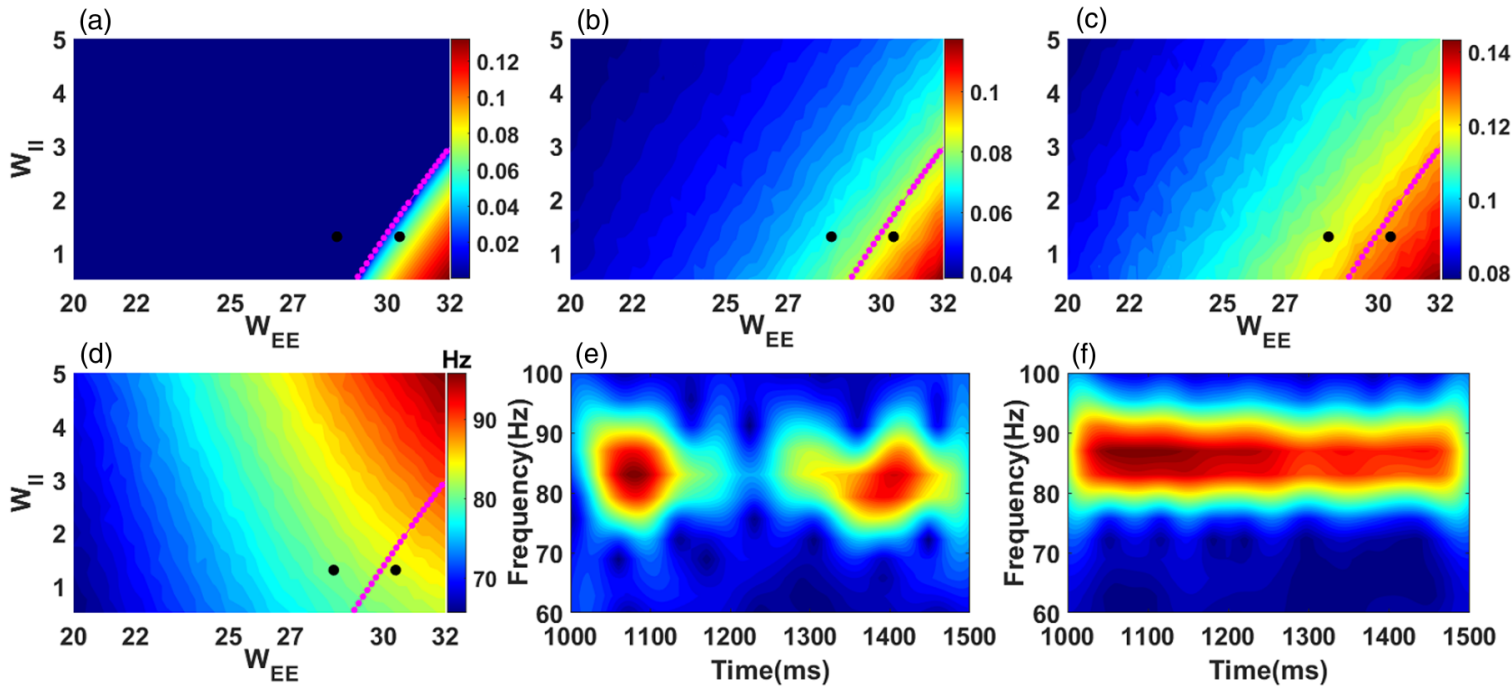

FIG. 1. Oscillations in the Stochastic Wilson-Cowan truncated to O(3), i.e., in the SWC3 model. (Top) Synchronization level of the $E$ cells in the subspace of self-connectivity parameters. The synchronization is measured from numerical simulations of the activities Eqs. (13)-(14) as the average difference between each trough and its successive peak. Specifically, we used a Gaussian filter and the Matlab function "findpeaks" to filter and extract the peaks and troughs of the LFPs. The curve in magenta represents the Hopf bifurcation boundary computed by setting the real part $-v$ of the complex conjugate eigenvalues to zero. The two black dots lie on either side of the Hopf boundary at $W_{I I}=1.3$ and $W_{E E}=27.4$ (quasicycle) or $W_{E E}=30.4$ (noisy limit cycle). (a) No noise: synchronization increases from zero beyond the Hopf bifurcation. (b) Weak noise $\left(\sigma_{E}=0.0015, \sigma_{I}=0.005, \sigma=0.005\right.$ ) causes "quasicycle" oscillations even below the Hopf bifurcation. (c) For stronger noise ( $\left.\sigma_{E}=0.004, \sigma_{I}=0.015, \sigma=0.0155\right)$, noise-induced oscillations are more prominent. (d) Frequency of the oscillations generated in (b). (e) Spectrogram corresponding to the black dot in the quasicycle regime in (b). Oscillations appear as discrete (short) epochs of synchrony called "bursts"; the frequency content also fluctuates from burst to burst. (f) Spectrogram for the black dot in the noisy limit-cycle regime in panel (b). Oscillations are now highly coherent with peak power around $f=88 \mathrm{~Hz}$. Parameters are $W_{E I}=26.3, W_{I E}=32, h_{E}=-3.8, h_{I}=-8, \alpha_{E}=$ $0.1, \alpha_{I}=0.2, \beta_{E}=1, \beta_{I}=2$. (d)-(f) use the noise intensities in panel (b). The parameters here are also used in Figs. 2 to 7 , unless otherwise stated.

The second equality leads to

$$
\lambda=\frac{1}{2}\left(A_{E E}+A_{I I}\right) \pm \frac{j}{2} \sqrt{-\left(A_{E E}-A_{I I}\right)^{2}-4 A_{E I} A_{I E}}
$$

We rewrite the eigenvalue in the compact form

$$
\lambda=-v \pm j \omega_{0}
$$

with

$$
\begin{aligned}
v & =-\frac{A_{E E}+A_{I I}}{2} \\
\omega_{0} & =\frac{1}{2} \sqrt{-\left(A_{E E}-A_{I I}\right)^{2}-4 A_{E I} A_{I E}} .
\end{aligned}
$$

This leads to the exact expression of the amplitude ratio between the $I$ and $E$ fluctuations for the linearized SWC3 system:

$$
\alpha=\left|\frac{\tilde{B}_{I}}{\tilde{B}_{E}}\right|=\frac{B_{I}}{B_{E}}=\sqrt{\frac{A_{I E}}{-A_{E I}}} .
$$

Similarly, the phase difference $\delta$ can be obtained from the relation

$$
\delta=\theta_{I}-\theta_{E}=\operatorname{Arg}\left(\frac{\tilde{B}_{I}}{\tilde{B}_{E}}\right) .
$$

For the parameters used here, we found

$$
\delta=\theta_{I}-\theta_{E}=\arctan \left(\frac{-2 \omega_{0}}{A_{E E}-A_{I I}}\right) .
$$

In Eqs. (16) and (17), $\|$ and Arg are respectively the modulus and the argument of the complex number $\frac{\tilde{B}_{I}}{\tilde{B}_{E}}$. In the presence of noise, one can compute amplitudes and phases of the $E$ and $I$ fluctuations from simulated time series using the analytic signal technique. The amplitude ratio and the phase difference are obtained by the following approximations:

$$
\alpha=\frac{B_{I}}{B_{E}} \approx\left\langle\frac{E n v\left[V_{I}(t)\right]}{E n v\left[V_{E}(t)\right]}\right\rangle
$$

and

$$
\delta=\theta_{I}-\theta_{E} \approx\left\langle\operatorname{Arg}\left[V_{I}(t)\right]-\operatorname{Arg}\left[V_{E}(t)\right]\right\rangle .
$$

Here $\langle$.$\rangle can be considered a time average of the stochastic$ process in Eqs. (13) and (14). Env is defined as the amplitude of the analytic signal associated with the LFP. For example, the analytic signal corresponding to $V_{E}(t)$ is $V_{E}(t)+$ $j H\left[V_{E}(t)\right]$, with the Hilbert transform $H$ defined as

$$
H[x]=\frac{1}{\pi} P \int_{-\infty}^{\infty} \frac{x(\tau)}{t-\tau} d \tau,
$$



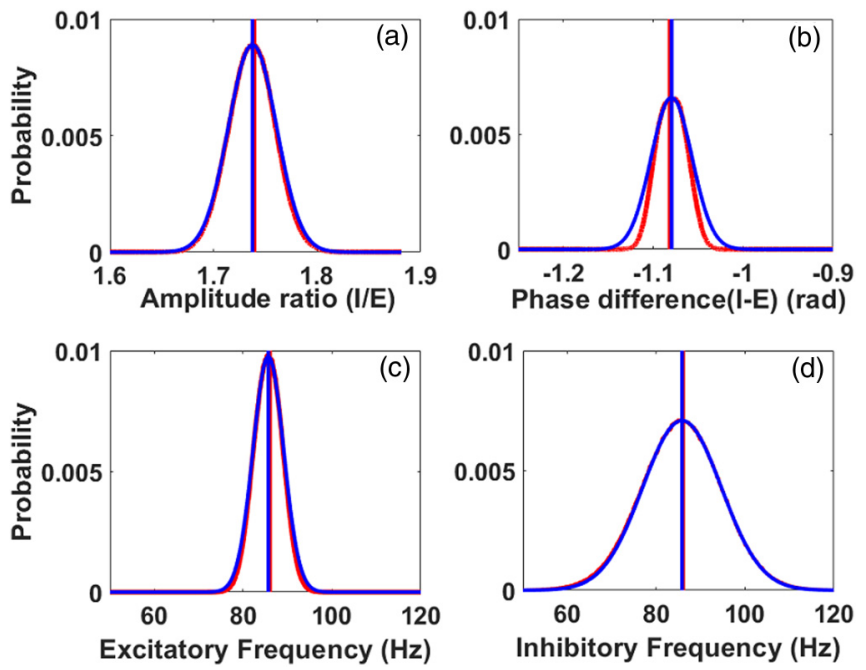

FIG. 2. $I / E$ amplitude ratio, $I-E$ phase difference and peak frequencies in the noisy limit-cycle regime (a) Probability distribution of the amplitude ratio between inhibitory and excitatory fluctuations from Eq. (16). (b) Probability distribution of the phase difference between inhibitory and excitatory fluctuations from Eq. (18). (c) Excitatory frequency probability distribution. (d) Inhibitory frequency probability distribution. For all the figures, (blue) curves correspond to the probability distributions computed numerically; the corresponding vertical lines are computed analytically from linear stability analysis Eqs. (16)-(18). The red curves are Gaussian fits to the blue curves, while the vertical red lines are the means of the red Gaussian distributions. Parameters are as in Fig. 1 with $W_{E E}=30.4$. Noise intensities are $\sigma_{E}=0.0002, \sigma_{I}=0.0006$, and $\sigma=0.0007$. Other parameters are as in Fig. 1.

where $P$ signifies the Cauchy principal value. The amplitude of the stochastic signal is then $\operatorname{Env}\left[V_{E}\right]=\sqrt{V_{E}^{2}+H^{2}\left[V_{E}\right]}$. Likewise, the phase angle of the analytic signal is defined as $\operatorname{Arg}\left[V_{E}\right]=\arctan \left[H\left[V_{E}\right] / V_{E}\right]$.

The transition between noise-induced and self-sustained oscillations happens when the real part of the eigenvalue is

$$
\begin{aligned}
& \text { zero. This condition is expressed as } \\
& \begin{aligned}
-\frac{\alpha_{E}}{1-E_{0}}+\alpha_{E} E_{0}\left[1-\frac{\alpha_{E} E_{0}}{\left(1-E_{0}\right) \beta_{E}}\right] W_{E E} & \frac{d Z_{E}}{d t}=F_{1}\left(Z_{E}, \phi_{E}\right)+G_{1}\left(Z_{E}, \phi_{E}, \eta_{E}, \eta_{I}\right), \\
-\frac{\alpha_{I}}{1-I_{0}}-\alpha_{I} I_{0}\left[1-\frac{\alpha_{I} I_{0}}{\left(1-I_{0}\right) \beta_{I}}\right] W_{I I}=0 . \quad(19) \quad \text { with the following functions: } & \frac{d \phi_{E}}{d t}=F_{2}\left(Z_{E}, \phi_{E}\right)+G_{2}\left(Z_{E}, \phi_{E}, \eta_{E}, \eta_{I}\right),
\end{aligned} \\
& \begin{aligned}
F_{1}\left(Z_{E}, \phi_{E}\right)= & \frac{1}{\alpha \sin (\delta)}\left[\alpha f_{1}\left(Z_{E}, \phi_{E}\right) \sin \left(\omega_{0} t+\phi_{E}+\delta\right)-f_{2}\left(Z_{E}, \phi_{E}\right) \sin \left(\omega_{0} t+\phi_{E}\right)\right], \\
F_{2}\left(Z_{E}, \phi_{E}\right)= & \frac{1}{\alpha Z_{E} \sin (\delta)}\left[\alpha f_{1}\left(Z_{E}, \phi_{E}\right) \cos \left(\omega_{0} t+\phi_{E}+\delta\right)-f_{2}\left(Z_{E}, \phi_{E}\right) \cos \left(\omega_{0} t+\phi_{E}\right)\right], \\
G_{1}\left(Z_{E}, \phi_{E}, \eta_{E}, \eta_{I}\right)= & \frac{1}{\alpha \sin (\delta)}\left[\alpha g_{1}\left(Z_{E}, \phi_{E}, \eta_{E}, \eta_{I}\right) \sin \left(\omega_{0} t+\phi_{E}+\delta\right)-g_{2}\left(Z_{E}, \phi_{E}, \eta_{E}, \eta_{I}\right) \sin \left(\omega_{0} t+\phi_{E}\right)\right], \\
G_{2}\left(Z_{E}, \phi_{E}, \eta_{E}, \eta_{I}\right)= & \frac{1}{\alpha Z_{E} \sin (\delta)}\left[\alpha g_{1}\left(Z_{E}, \phi_{E}, \eta_{E}, \eta_{I}\right) \cos \left(\omega_{0} t+\phi_{E}+\delta\right)-g_{2}\left(Z_{E}, \phi_{E}, \eta_{E}, \eta_{I}\right) \cos \left(\omega_{0} t+\phi_{E}\right)\right], \\
f_{1}\left(Z_{E}, \phi_{E}\right)= & \omega_{0} Z_{E} \sin \left(\omega_{0} t+\phi_{E}\right)+A_{E E} Z_{E} \cos \left(\omega_{0} t+\phi_{E}\right)+A_{E I} \alpha Z_{E} \cos \left(\omega_{0} t+\phi_{E}+\delta\right) \\
& +L_{E E} Z_{E}^{2} \cos ^{2}\left(\omega_{0} t+\phi_{E}\right)+L_{E I} \alpha Z_{E}^{2} \cos \left(\omega_{0} t+\phi_{E}\right) \cos \left(\omega_{0} t+\phi_{E}+\delta\right)+B_{1 E} Z_{E}^{3} \cos { }^{3}\left(\omega_{0} t+\phi_{E}\right) \\
& +B_{2 E} \alpha^{2} Z_{E}^{3} \cos \left(\omega_{0} t+\phi_{E}\right) \cos ^{2}\left(\omega_{0} t+\phi_{E}+\delta\right)+B_{3 E} \alpha Z_{E}^{3} \cos { }^{2}\left(\omega_{0} t+\phi_{E}\right) \cos \left(\omega_{0} t+\phi_{E}+\delta\right),
\end{aligned}
\end{aligned}
$$

where $Z_{E}$ and $\phi_{E}$ represent the stochastic amplitude and phase the $E$ fluctuations. The idea is to compute the dynamics of $Z_{E}$ and the phase $\phi_{E}$ of the $E$ fluctuations. The paramers $\alpha, \delta$, and $\omega_{0}$ are the amplitude ratio, phase difference, Eqs. (16)-(18) (see Fig. 2). We then insert these expressions into Eqs. (13) and (14) and obtain 


$$
\begin{aligned}
f_{2}\left(Z_{E}, \phi_{E}\right)= & \alpha \omega_{0} Z_{E} \sin \left(\omega_{0} t+\phi_{E}+\delta\right)+A_{I E} Z_{E} \cos \left(\omega_{0} t+\phi_{E}\right)+A_{I I} \alpha Z_{E} \cos \left(\omega_{0} t+\phi_{E}+\delta\right)+L_{I I} \alpha^{2} Z_{E}^{2} \\
& \times \cos ^{2}\left(\omega_{0} t+\phi_{E}+\delta\right)+L_{I E} \alpha Z_{E}^{2} \cos \left(\omega_{0} t+\phi_{E}\right) \cos \left(\omega_{0} t+\phi_{E}+\delta\right)+B_{1 I} \alpha^{3} Z_{E}^{3} \cos ^{3}\left(\omega_{0} t+\phi_{E}+\delta\right) \\
& +B_{2 I} \alpha^{2} Z_{E}^{3} \cos \left(\omega_{0} t+\phi_{E}\right) \cos ^{2}\left(\omega_{0} t+\phi_{E}+\delta\right)+B_{3 I} \alpha Z_{E}^{3} \cos ^{2}\left(\omega_{0} t+\phi_{E}\right) \cos \left(\omega_{0} t+\phi_{E}+\delta\right), \\
g_{1}\left(Z_{E}, \phi_{E}, \eta_{E}, \eta_{I}\right)= & \eta_{E}(t), \quad g_{2}\left(Z_{E}, \phi_{E}, \eta_{E}, \eta_{I}\right)=\eta_{I}(t)
\end{aligned}
$$

However, such equations are cumbersome and difficult to analyze since the amplitude and phase are coupled in a complicated manner. We need simpler equations for a suitable analysis of amplitude dynamics, which we now determine.

\section{Stochastic averaging method (SAM)}

To simplify the amplitude-phase dynamics in Eqs. (21) and (22), we make use of the stochastic averaging method (SAM) $[72,82]$, which stipulates that the system can be approximated by a two-dimensional Markov process given by

$$
d\left(\begin{array}{l}
Z_{E}(t) \\
\phi_{E}(t)
\end{array}\right)=\left(\begin{array}{l}
m_{1}(t) \\
m_{2}(t)
\end{array}\right) d t+\left(\begin{array}{ll}
h_{11}(t) & h_{12}(t) \\
h_{21}(t) & h_{22}(t)
\end{array}\right)\left(\begin{array}{l}
d W_{1}(t) \\
d W_{2}(t)
\end{array}\right)
$$

with

$$
\begin{aligned}
& m_{i}(X)=T^{a v}\left(E\left\{F_{i}\right\}+\sum_{j} \int_{-\infty}^{0} E\left\{\left(\frac{\partial G_{i}}{\partial X_{j}}\right)_{t}\left(G_{j}\right)_{t+\tau}\right\} d \tau\right), \\
& {\left[h h^{\prime}\right]_{i j}=T^{a v}\left(\int_{-\infty}^{\infty} E\left\{\left(G_{i}\right)_{t}\left(G_{j}\right)_{t+\tau}\right\} d \tau\right), \quad i, j=1,2 .}
\end{aligned}
$$

Here $m=\left(\begin{array}{c}m_{1}(t) \\ m_{2}(t)\end{array}\right), F=\left(\begin{array}{c}F_{1}(t) \\ F_{2}(t)\end{array}\right), G=\left(\begin{array}{c}G_{1}(t) \\ G_{2}(t)\end{array}\right), X=\left(\begin{array}{c}Z_{E}(t) \\ \phi_{E}(t)\end{array}\right), h$ is the matrix made up of the elements $h_{i j},\left(h h^{\prime}\right)$ is the product of $h$ and its transpose.

Moreover, $E$. denotes the expectation operator (i.e. average over realizations), and $T^{a v}$ is the time averaging operator defined by

$$
T^{a v}(.)=\frac{1}{T_{0}} \int_{t_{0}}^{t_{0}+T_{0}}(.) d t .
$$

The subscript $t$ and $t+\tau$ mean that the elements of the corresponding vectors and matrix are evaluated at these times. The SAM further assumes that the two-dimensional functions $F\left(Z_{E}, \phi_{E}\right)$ and $G\left(Z_{E}, \phi_{E}, \xi_{E}, \xi_{I}\right)$ are respectively of order $\mathrm{O}(\epsilon)$ and $\mathrm{O}\left(\epsilon^{2}\right)$, where $\epsilon$ is a small parameter. The averaging in the SAM procedure assumes that the functions $Z_{E}(t)$ and $\phi_{E}(t)$ are constant during the period $T_{0}=2 \pi / \omega_{0}$ of the underlying deterministic oscillation $\left(\omega_{0}\right.$ also varies with system parameters). After some calculations, we end up with the following amplitude-phase dynamics:

$$
\begin{gathered}
d Z_{E}=\left[-v Z_{E}+B_{1} Z_{E}^{3}+\frac{D}{2 Z_{E}}\right] d t+\sqrt{D} d W_{1}, \\
d \phi_{E}=B_{2} Z_{E}^{2} d t+\frac{\sqrt{D}}{Z_{E}} d W_{2} .
\end{gathered}
$$

If we further make the change of variable $\theta_{E}(t)=\omega_{0} t+$ $\phi_{E}(t)$, the dynamics of the fast phase $\theta_{E}(t)$ is obtained from Eq. (24) as

$$
d \theta_{E}=\left[\omega_{0}+B_{2} Z_{E}^{2}\right] d t+\frac{\sqrt{D}}{Z_{E}} d W_{2}
$$

with the following expressions:

$$
\begin{aligned}
B_{1}= & \frac{1}{8}\left[3 B_{1 E}+B_{3 I}+\alpha^{2}\left(B_{2 E}+3 B_{1 I}\right)\right. \\
& \left.+2 \alpha \cos (\delta)\left(B_{3 E}+B_{2 I}\right)\right] \\
B_{2}= & \frac{1}{8 \sin (\delta)}\left[2 \alpha\left(B_{3 E}-B_{2 I}\right)+3\left(B_{1 E}-B_{3 I}\right) \cos (\delta)\right. \\
& \left.+3 \alpha^{2}\left(B_{2 E}-B_{1 I}\right) \cos (\delta)+\alpha\left(B_{3 E}-B_{2 I}\right) \cos (2 \delta)\right] \\
D= & \frac{1}{2 \alpha^{2} \sin ^{2}(\delta)}\left[\frac{2 \tau_{E}\left(\alpha \sigma_{E}\right)^{2}}{1+\left(\omega_{0} \tau_{E}\right)^{2}}+\frac{2 \tau_{I}\left(\sigma_{I}\right)^{2}}{1+\left(\omega_{0} \tau_{I}\right)^{2}}\right]
\end{aligned}
$$

Here $W_{1}(t)$ and $W_{2}(t)$ are two independent Wiener processes. The coefficient $B_{1}$ is negative for the parameters used here, which implies that the amplitude $Z_{E}$ remains finite, i.e., deviations from the fixed point remain bounded. Taking into account the power spectra at $\omega_{0}$,

$$
S_{\eta_{E, I}}\left(\omega_{0}\right)=\frac{2 \tau_{E, I}\left(\sigma_{E, I}\right)^{2}}{2 \pi\left[1+\left(\omega_{0} \tau_{E, I}\right)^{2}\right]},
$$

of the OU processes $\eta_{E}(t)$ and $\eta_{I}(t)$ applied respectively on the $E$ and $I$ dynamics, we find that the effective noise strength $D$ of the amplitude-phase dynamics is a weighted sum of these power spectra:

$$
D=\frac{\pi}{\alpha^{2} \sin ^{2}(\delta)}\left[\alpha^{2} S_{\eta_{E}}\left(\omega_{0}\right)+S_{\eta_{I}}\left(\omega_{0}\right)\right] .
$$

Different noise processes can be handled by substituting the proper power spectra in this expression for $D$.

Most of our simulations in this work consider additive Gaussian white noise on the SWC3 model Eqs. (13) and (14) and further below on the SSL model, although we do later explore the effect of the noise correlation time in the SSL model in Figs. 10 and 11. For the white noise case $\eta_{E, I}(t)=$ $\sigma_{E, I} \xi_{E, I}(t)$, the above amplitude-phase dynamics apply with the definition of $D$ based on the white noise power spectra $S\left(\omega_{0}\right)=\frac{\sigma_{E, I}^{2}}{2 \pi}$, that is,

$$
D=\frac{\left(\sigma_{I}\right)^{2}+\left(\alpha \sigma_{E}\right)^{2}}{2 \alpha^{2} \sin ^{2}(\delta)} .
$$

Further, since the noise is only additive, there is no need to distinguish between Itô and Stratonovich calculus; the SAM method itself was developed using Itô calculus $[72,82]$.

As for the case of the quasicycle oscillations [60], the amplitude dynamics is uncoupled from the phase dynamics. In contrast however, a new term $B_{1} Z_{E}^{3}$ appears in the amplitude dynamics. This term is at the origin of the stabilization of the growth of the amplitude when the real part of the complex conjugate eigenvalues becomes positive beyond the Hopf bifurcation. The phase dynamics also has a new deterministic term $B_{2} Z_{E}^{2}$. This causes the deterministic frequency to depend 

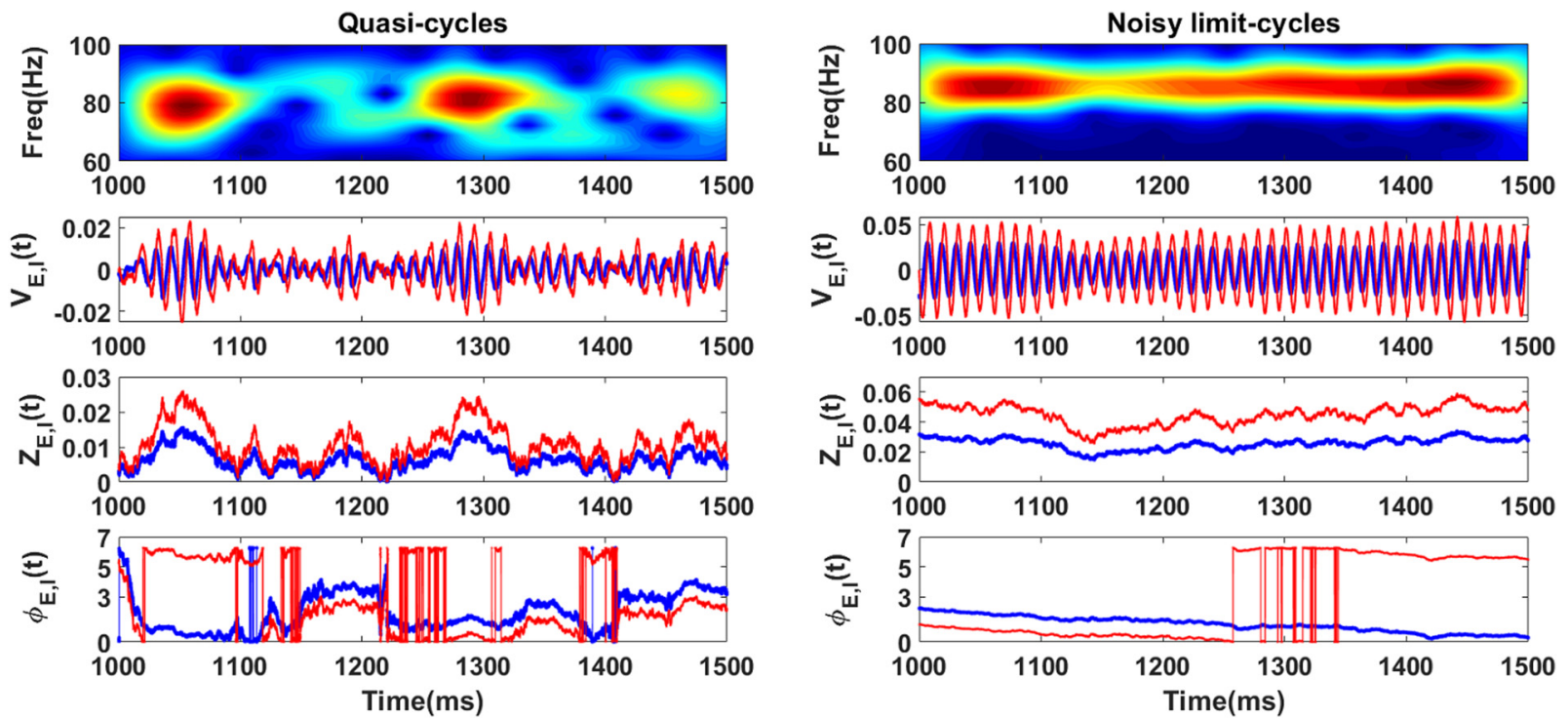

FIG. 3. Quasicycle and noisy limit-cycle properties using the SAM approximation. From top to bottom: Spectrogram, activity timeseries, amplitudes and phases of the excitatory (blue) and inhibitory (blue) populations simulated using the dynamics Eqs. (23) and (24) computed using the SAM analysis. Left: quasicycle regime with its characteristic bursting structure. Fluctuations, amplitudes and phases resemble dynamics obtained with linear equations driven by noise [59,60]. (Right) Self-sustained oscillations perturbed by noise, with almost constant envelope, phase and frequency characteristic of noisy limit cycles. Quasicycle parameters are as for the left black dot in Fig. 1(b), i.e., $W_{E E}=$ 27.4. Limit cycle parameters are as for the right black dot in Fig. 1(b), i.e., $W_{E E}=30.4$. The noise strengths are $\sigma_{E}=0.0015, \sigma_{I}=0.005, \sigma=$ 0.005. Other parameters are as in Figs. 1(e) and 1(f).

on the amplitude magnitude. This contrasts with the quasicycle regime obtained with the linear WC system, where the term $B_{2}$ was absent.

Next, we compute spectrograms and time-series of the fluctuations $V_{E, I}(t)$, their amplitudes $Z_{E, I}(t)$ and their phases $\phi_{E, I}(t)$ both for the quasicycle and noisy limit-cycle regimes (Fig. 3). We observe that our amplitude-phase representation describes well the dynamics of $E$ and $I$ fluctuations for both quasicycles (left panels) and noisy limit cycles (right panels). Our model can thus account for both noise-induced oscillations and noise-perturbed oscillations. It produces bursting epochs for quasicycles and self-sustained oscillations of long duration for the noisy limit cycles.

\section{Deterministic analysis of the amplitude dynamics}

We focus on the deterministic case $D=0$, for which the amplitude dynamics are

$$
\frac{d Z_{E}^{0}}{d t}=-v Z_{E}^{0}+B_{1}\left(Z_{E}^{0}\right)^{3}
$$

Let us define $F_{0}\left(Z_{E}\right) \equiv-v Z_{E}+B_{1}\left(Z_{E}\right)^{3}$ and its derivative $F_{0}^{\prime}\left(Z_{E}\right)=-v+3 B_{1}\left(Z_{E}\right)^{2}$. The solutions of $F\left(Z_{E}^{0}\right)=0$ are the fixed points of the deterministic amplitude dynamics Eq. (29). Their stability is given by the sign of the corresponding value of $F^{\prime}\left(Z_{E}^{0}\right)$. The existence and the number of the solutions of $F\left(Z_{E}^{0}\right)=0$ depend on whether the system lies below or above the Hopf bifurcation.

(1) Stable focus $(v>0)$. The only fixed point is $Z_{E}^{0}=0$. This fixed point is stable since $F^{\prime}(0)=-v<0$. This is in agreement with previous results, since in the deterministic limit and below the Hopf bifurcation, the amplitude of a perturbation from the fixed point converges to zero.

(2) Limit-cycle $(v<0)$ : We have two fixed points $Z_{E}^{0}=0$ and $Z_{E}^{0}=\sqrt{\frac{\nu}{B_{1}}}$. But the only stable fixed point is $Z_{E}^{0}=\sqrt{\frac{\nu}{B_{1}}}$ since $F^{\prime}(0)=-v>0$ and $F^{\prime}\left(\sqrt{\frac{v}{B_{1}}}\right)=2 v<0$. The value of this fixed point represents the amplitude of the oscillation. The size of the limit cycle thus varies as $\sqrt{v}$ as expected from the normal form of the supercritical Hopf bifurcation [78].

Now, we turn to the effect of the noise on this deterministic picture.

\section{Stochastic analysis of the amplitude dynamics $(D>0)$}

To perform the stochastic analysis of the amplitude process, we consider the corresponding stationary Fokker-Planck equation of (23) [79]:

$$
-\frac{d}{d Z_{E}}\left[\left(-v Z_{E}+\frac{D}{2 Z_{E}}+B_{1} Z_{E}^{3}\right) P_{E}\right]+\frac{D}{2} \frac{d^{2} P_{E}}{d Z_{E}^{2}}=0,
$$

where $P_{E}\left(Z_{E}\right)$ represents the stationary probability density function of the amplitude process $Z_{E}(t)$. We define the following parameters:

$$
a=\sqrt{\frac{-B_{1}}{2 D}} \text { and } b=\frac{-v}{B_{1}} .
$$

The normalized solution $\left(\int_{0}^{\infty} P_{E}\left(Z_{E}\right)=1\right)$ of this second order differential equation is given by

$$
P_{E}\left(Z_{E}\right)=\frac{4 a}{\sqrt{\pi} \operatorname{erfc}(b a)} Z_{E} \exp \left[-a^{2}\left(Z_{E}^{2}+b\right)^{2}\right] .
$$


By using the relation between the amplitude of excitatory and inhibitory LFPs $\left(Z_{I}=\alpha Z_{E}\right)$, we obtain the stationary probability density function for the inhibitory amplitude process:

$$
P_{I}\left(Z_{I}\right)=\frac{4 a}{\alpha^{2} \sqrt{\pi} \operatorname{erfc}(b a)} Z_{I} \exp \left[-\left(\frac{a}{\alpha^{2}}\right)^{2}\left(Z_{I}^{2}+\alpha^{2} b\right)^{2}\right],
$$

where $\operatorname{erfc}($.$) denotes the complementary error function de-$ fined as $\operatorname{erfc}(x)=\frac{2}{\sqrt{\pi}} \int_{x}^{\infty} e^{-t^{2}} d t$. The peak of the stationary density, which represents the most probable value taken by the stochastic amplitude process, is given by $\frac{d P_{E}\left(Z_{E}\right)}{d Z_{E}}=0$.

This relation leads to the following equation:

$$
1-4 a^{2} b Z_{E}^{2}-4 a^{2} Z_{E}^{4}=0 .
$$

The solution of this equation can be obtained for the two distinct regimes.

(1) Quasicycle regime $(v>0, \quad b>0)$. The solution is given by

$$
Z_{E}^{2}=-\frac{b}{2}+\frac{b}{2}\left(1+\frac{1}{(a b)^{2}}\right)^{1 / 2} .
$$

The approximate expressions for the location of the peaks of the $E$ and $I$ amplitude probability densities are then

$$
Z_{E}^{*} \approx \sqrt{\frac{D}{2 v}} \text { and } Z_{I}^{*} \approx \alpha \sqrt{\frac{D}{2 v}} .
$$

We observe that the peak values of the stationary probability densities are in agreement with the previous results reported for the linearized Wilson-Cowan equations driven by additive noise [60]. Moreover, in the limit $B_{1}=0$ using $b \rightarrow \infty, a \rightarrow$ $0, a b \rightarrow \infty$, the $E$ stationary probability density can be written as (we drop the subscript $E$ for the sake of readability):

$$
\begin{aligned}
P_{E}(Z) & =\frac{4 a}{\operatorname{erfc}(b a) \sqrt{\pi}} Z \exp \left[-a^{2}\left(Z^{2}+b\right)^{2}\right] \\
& =\frac{4 a}{\sqrt{\pi}}(1-\operatorname{erf}(b a))^{-1} Z e^{-(a b)^{2}} e^{-a^{2} Z^{4}} e^{-2 b a^{2} Z^{2}} \\
& \approx \frac{4 a}{\sqrt{\pi}}(1-\operatorname{erf}(b a))^{-1} Z e^{-(a b)^{2}} e^{-2 b a^{2} Z^{2}} .
\end{aligned}
$$

Expanding the error function as

$$
\operatorname{erf}(a b) \approx 1-e^{-(a b)^{2}} \frac{1}{\sqrt{\pi}}\left(\frac{1}{a b}-\frac{1}{2(a b)^{3}}+\ldots\right),
$$

we then obtain

$$
\begin{aligned}
P_{E}(Z) & \approx \frac{4 a}{\sqrt{\pi}}\left(e^{-(a b)^{2}} \frac{1}{\sqrt{\pi}} \frac{1}{a b}\right)^{-1} Z e^{-(a b)^{2}} e^{-2 b a^{2} Z^{2}} \\
& \approx 4 a^{2} b Z \exp \left(-\frac{v}{D} Z^{2}\right) .
\end{aligned}
$$

We thus recover the expressions of the probability densities obtained in the case of linear equations driven by additive noise [60], namely,

$$
P_{E}\left(Z_{E}\right)=\frac{2 v}{D} Z_{E} \exp \left(-\frac{v}{D} Z_{E}^{2}\right)
$$

for the excitatory amplitude process and

$$
P_{I}\left(Z_{I}\right)=\frac{2 v}{\alpha^{2} D} Z_{I} \exp \left(-\frac{v}{\alpha^{2} D} Z_{I}^{2}\right)
$$

for the inhibitory amplitude process.

(2) Noisy limit-cycle regime $(v<0, \quad b<0)$. The solution is given by

$$
Z_{E}^{2}=-\frac{b}{2}-\frac{b}{2}\left(1+\frac{1}{(a b)^{2}}\right)^{1 / 2} .
$$

This leads to the following approximate expressions obtained in weak noise limit:

$$
Z_{E}^{*} \approx \sqrt{\frac{v}{B_{1}}+\frac{D}{2(-v)}} \text { and } Z_{I}^{*} \approx \alpha \sqrt{\frac{v}{B_{1}}+\frac{D}{2(-v)}} .
$$

We should mention that the approximations in Eqs. (35) and (38) are valid for weak noise and away from the Hopf bifurcation. They were derived to get an idea of the role of noise on the limit-cycle dynamics. The accurate expression for the peak of the amplitude probability density is determined by Eq. (33).

We see that for $D=0$, we recover the result previously obtained in the deterministic analysis. Importantly, for $D>0$ the most probable amplitude value for the noisy limit cycle is greater than that for the deterministic case. There is an additional term which is proportional to the strength of the noise $D$ and inversely proportional to the real part of the eigenvalues. This is a noise-induced term which shows how noise shapes the amplitude of the limit cycle by increasing its magnitude.

We plot the densities of the $E$ and $I$ amplitude processes for the quasicycles and noisy limit cycles in Figs. 4(a), 4(c) and 4(b), 4(d) respectively. We find an excellent match between analytical expressions from Eqs. (31) and (32) (black curves) and numerical simulations of Eqs. (13) and (14) (magenta curves). Also we found excellent agreement with the values of the peaks of the pdf (vertical blue lines) computed from the stochastic analysis Eqs. (38) in both regimes.

In the following, we mostly focus on the dynamics of the noisy limit cycle since the case of the quasicycle has already been investigated in a previous study [60], and the results here with the extra nonlinearity are qualitatively the same. Can strong noise induce bursting structures in the limit-cycle regime like those seen for quasicycles? If so, how can we discriminate between these two dynamical origins for the burst epochs?

\section{Dynamics of the noisy limit cycle}

We investigate the effect that different noise strengths can have on the amplitudes of limit-cycle oscillations. We consider the limit-cycle regime close to the supercritical Hopf bifurcation (black dot, Fig. 1), where the system is weakly nonlinear and the amplitude of the deterministic limit cycle is small. The amplitude density shows little variation with noise strength in the weak noise limit. The corresponding probability densities in Figs. 5(a) and 5(b), red curves, are close to Gaussian (by visual inspection) with small variance; their peaks can be predicted from the theoretical expressions in Eqs. (33) and (38). 

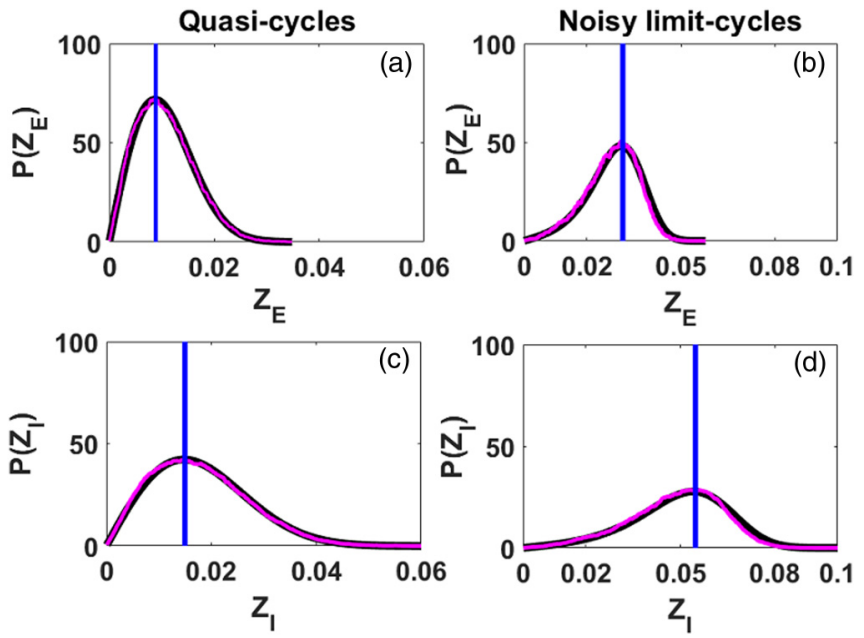

FIG. 4. Probability densities of excitatory and inhibitory amplitude processes. (Left) Probability densities of excitatory (top) and inhibitory (bottom) amplitude processes in the quasicycle regime. (Right) Probability densities of excitatory (top) and inhibitory (bottom) amplitude processes in the noisy limit-cycle regime. The black curves correspond to analytical results [Eqs. (31) and (32)] and the magenta curves are from numerical simulations of Eqs. (13) and (14). The vertical blue lines are analytical values of the probability density peak locations computed from Eq. (35) for the quasicycle (left) and from Eq. (38) for the limit-cycle regimes (right). Theory matches up very well with numerical simulations. We also note a transition of the density shape from Rayleigh in the quasicycle case to approximately Gaussian in the noisy limit-cycle case. Quasicycles [(a) and (c)]: $W_{E E}=27.4$. Noisy limit cycles $\left[(\mathrm{b})\right.$ and (d)]: $W_{E E}=30.4$. Noise strengths are $\sigma_{E}=0.0015, \sigma_{I}=0.005, \sigma=0.005$, and other parameters are as in Fig. 1.

The small variance causes long segments of uninterrupted strong oscillations, i.e., minimal bursting, in contrast to what is observed for the quasicycles. As we further increase the noise strength, the peak and variance of the amplitude values increase. For strong noise [Figs. 5(a) and 5(b), blues curves], the peak amplitude values are high with corresponding large variance. The fact that amplitude values near zero are now more probable compared to the weak noise limit suggests that bursts are favored under these conditions. To unveil the interplay between the noise and the size of the limit cycle, we now increase the size of the limit cycle using larger recurrent excitation strengths $W_{E E}$ as suggested by Fig. 1, as well as vary the noise strength.

For weak noise in Figs. 6(a) and 6(b), increasing $W_{E E}$ increases the peak value of the oscillation amplitude and decreases its variance. This suggests that bursting decreases moving further beyond the bifurcation. Intermediate noise in Figs. 6(c) and 6(d) increases the variance, thus causing bursts, as is seen for all limit-cycle sizes over this range of $W_{E E}$. The effect of noise on the peak amplitude is more significant close to the Hopf bifurcation. Strong noise in Figs. 6(e) and 6(f) increases variance for all limit-cycle sizes, but increases the peak mostly near the Hopf bifurcation. This can be understood from Eqs. (38). Close to the Hopf bifurcation, $|v|$ is small and the effect of the term $\frac{D}{2(-\nu)}$ dominates. Far from the bifurcation however, $|\nu|$ is larger and $\frac{D}{2(-v)}$ is now less important. The
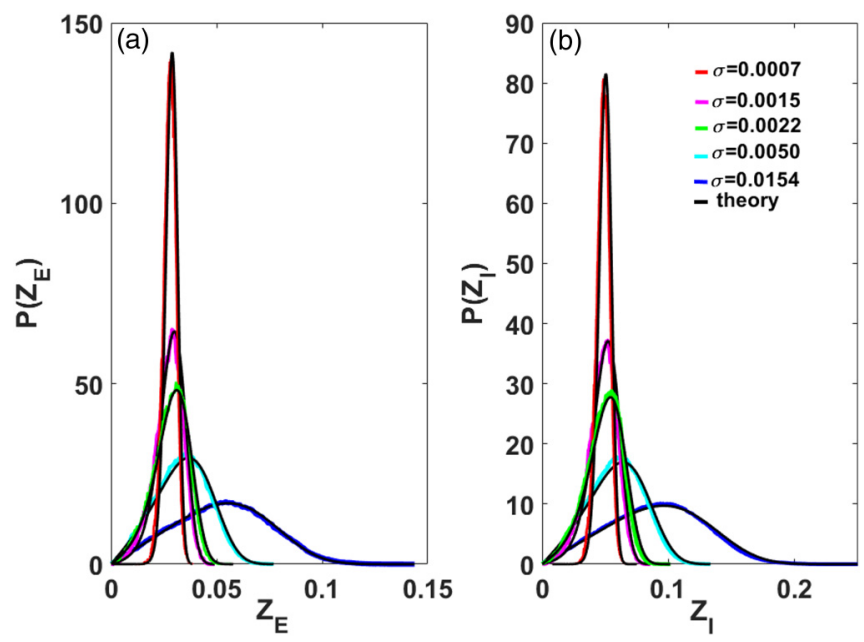

FIG. 5. Effect of noise on the limit-cycle oscillations. We consider the limit-cycle regime just beyond the Hopf bifurcation (see Fig. 1) and look at the effect of the noise strength on the dynamics of the $E$ (a) and $I$ (b) amplitudes. High noise increases the overall amplitude values but also increases their variability, enabling oscillatory bursts. Black curves correspond to theory while other colors correspond to numerical simulations at different noise strengths as specified in the legend to panel (b). In detail, blue: $\sigma_{E}=$ $0.004, \quad \sigma_{I}=0.015$, and $\sigma=0.0154 ;$ cyan: $\sigma_{E}=0.0015, \quad \sigma_{I}=$ 0.005 , and $\sigma=0.005$; green: $\sigma_{E}=0.0006, \sigma_{I}=0.002$, and $\sigma=$ 0.0022 ; magenta: $\sigma_{E}=0.00045, \sigma_{I}=0.0015$, and $\sigma=0.0015$; red: $\sigma_{E}=0.0002, \sigma_{I}=0.0006$, and $\sigma=0.0007$. In all cases, there is good agreement between theory and numerics.
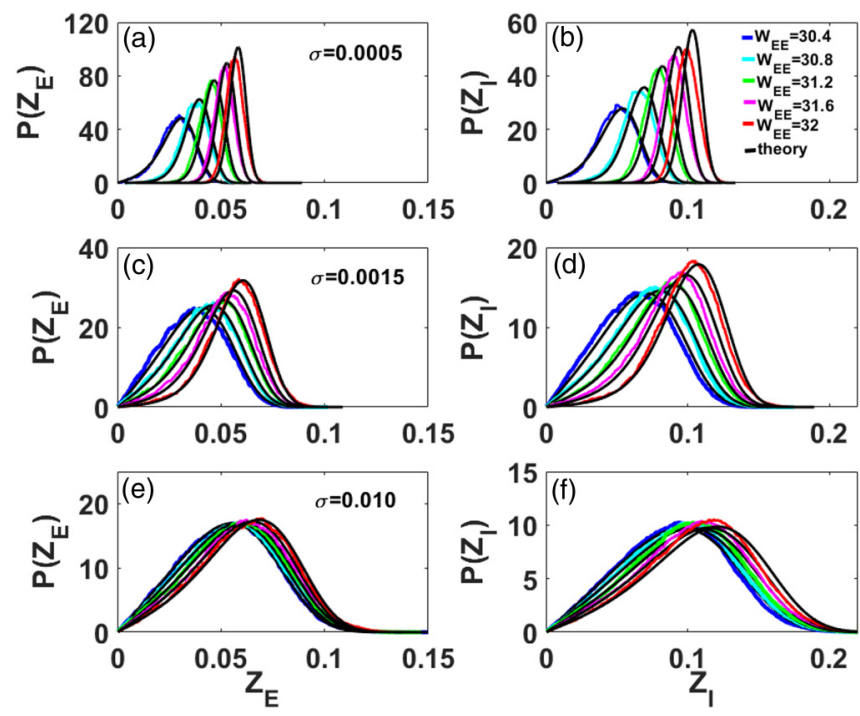

FIG. 6. Interplay between noise and limit-cycle amplitude density. For weak noise [(a) and (b)], moving beyond the Hopf bifurcation by increasing $W_{E E}$ leads to an increase in the peak amplitude and a reduction of its variability. For intermediate noise [(c) and (d)], an increase in variability is visible for all values of $W_{E E}$. The effect of noise strength on increasing the peak amplitude of the oscillations is prominent near the Hopf bifurcation (weak values of $W_{E E}$ ), but very weak far from it. For strong noise [(e) and (f)], the amplitude peak and variability increase again for all $W_{E E}$. Other parameters are as in Fig. 1. 

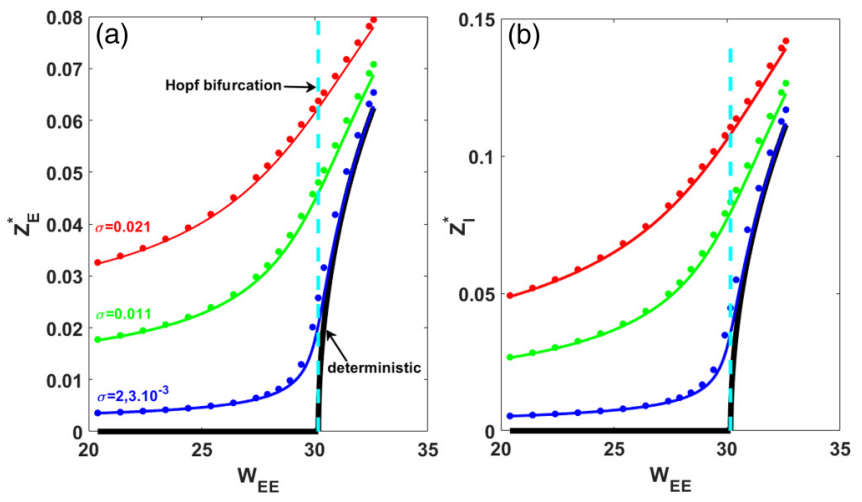

FIG. 7. Bifurcation diagram of the SWC3 model. Peaks of the probability densities of the $E$ (a) and $I$ (b) amplitude fluctuations as a function of the recurrent excitatory coupling. Noise smoothes the sharp Hopf bifurcation and increases the peak amplitude, i.e. it induces the quasicycles. However, these densities does not reveal the regularity or coherence of the oscillation. For all the curves, the solid lines correspond to the theoretical expression of the peak amplitude, while the dots are the result of numerical simulation which are in good agreement with the SAM theory. Other parameters are as in Fig. 1.

peak amplitude thus depends less on $D$ and instead varies mostly as $\frac{\nu}{B_{1}}$.

We next directly characterize the effect of noise on the peak of the probability density given by the solution of Eq. (33). This can now be done for both the quasi- and limit-cycle regimes. Figure 7 shows that increasing noise strength produces more smoothing of the bifurcation and higher most probable values. The results computed by the SAM are in good agreement with numerical simulations obtained through the Hilbert transform performed on the SWC3 dynamics. This speaks to the predictive power of the SAM technique in this system, as it provides a theoretical handle on amplitude strength as a function of the intrinsic network parameters and the external noise. The most probable amplitude value is also an indirect measure of level of network synchronization, and can be seen as an order parameter for the stochastic bifurcation as Fig. 7 reveals.

Our analysis also shows that noise shapes the amplitude and burstiness of the limit-cycle amplitude. In particular, strong noise can create bursts even in the limit-cycle regime. Quasicycle bursts are a signature of an induced oscillation, but in the limit-cycle regime, they are a sign of strong noise. It is difficult to differentiate between these two cases just by looking at the spectrograms. However, this can be helped by also considering the envelope probability densities. If the density is closer to Rayleigh than Gaussian, the spectrogram is likely the result of quasicycle dynamics; a better fit to a Gaussian points instead to a noisy limit cycle.

\section{STOCHASTIC STUART-LANDAU MODEL}

To assess the generality of our amplitude-phase decomposition across the bifurcation, we now turn to the stochastic Stuart-Landau (SSL) model [94]. Its deterministic dynamics is the canonical form of the supercritical Hopf bifurcation. We perform an amplitude-phase decomposition and pay particular attention to the noisy limit-cycle regime and the effect of correlated noise. The dynamics is given as

$$
\begin{aligned}
& \dot{x}(t)=a_{0} x(t)-\omega_{0} y(t)-x(t)\left[x^{2}(t)+y^{2}(t)\right]+\eta_{x}(t), \\
& \dot{y}(t)=\omega_{0} x(t)+a_{0} y(t)-y(t)\left[x^{2}(t)+y^{2}(t)\right]+\eta_{y}(t) .
\end{aligned}
$$

The eigenvalues around the origin are given by $\lambda=a_{0} \pm j \omega_{0}$, and the supercritical Hopf bifurcation happens at $a_{0}=0$. Quasicycles exist in the region $\left(a_{0}<0 ; \omega_{0}>0\right)$ and noisy limit cycles are defined in the region $\left(a_{0}>0 ; \omega_{0}>0\right)$. We proceed as in the case of SWC3 equations. We first separate the dynamics of the model into its fixed point and the corresponding fluctuations as $x(t)=x_{0}+V_{x}(t)$ and $y(t)=y_{0}+V_{y}(t)$. Note that for the Stuart-Landau model, the fixed point is the origin $\left(x_{0}=y_{0}=0\right)$. However, we will keep the notations $x_{0}$ and $y_{0}$ to easily make the connection with the analysis done in the SWC3 model. By identification with the analysis done in the case of the SWC3 system in Eqs. (13) and (14), we obtain the following coefficients for the dynamics of the fluctuations (with subscripts $E$ and $I$ replaced by $x$ and $y$, respectively):

$$
\begin{aligned}
A_{x x} & =a_{0}-\left(3 x_{0}^{2}+y_{0}^{2}\right), \\
A_{y y} & =a_{0}-\left(x_{0}^{2}+3 y_{0}^{2}\right), \\
A_{x y} & =-\left(\omega_{0}+2 x_{0} y_{0}\right), \\
A_{y x} & =\omega_{0}-2 x_{0} y_{0}, \\
L_{x x} & =-3 x_{0}, \quad L_{x y}=-2 y_{0}, \\
L_{y x} & =-2 x_{0}, \quad L_{y y}=-3 y_{0}, \\
M_{x y} & =-x_{0}, \quad M_{y x}=-y_{0}, \\
B_{1 x} & =-1, \quad B_{2 x}=-1, \\
B_{3 x} & =0, \quad B_{1 y}=-1, \\
B_{2 y} & =0, \quad B_{3 y}=-1, \\
B_{4 x} & =0 ; \quad B_{4 y}=0 .
\end{aligned}
$$

The fixed amplitude ratio and phase difference between $x$ and $y$ components are also obtained similarly, with $\omega_{o}$ again considered as the oscillation frequency. However, in the case of the SSL model, the amplitude computed through linear stability analysis is $\alpha=1$ [Fig. 8(a)] and the phase difference is $\delta=-\frac{\pi}{2}$ [Fig. 8(b)]. Therefore the solution can be sought in the form

$$
V_{x}=Z(t) \cos \left(\omega_{0} t+\phi(t)\right), \quad V_{y}=Z(t) \sin \left(\omega_{0} t+\phi(t)\right) .
$$

We have assumed $Z_{x}=Z$ for readability. We then applied the SAM using the above expressions and obtained

$$
\begin{gathered}
d Z(t)=\left[a_{0} Z(t)-Z^{3}(t)+\frac{D}{2 Z(t)}\right] d t+\sqrt{D} d W_{1}(t), \\
d \phi(t)=B_{2} Z^{2}(t) d t+\frac{\sqrt{D}}{Z(t)} d W_{2}(t) .
\end{gathered}
$$

For the case of the SSL model, the coefficient $B_{1}=-1$ and $B_{2}=0$. We choose to replace $B_{1}$ by its value to show the specificity of the SSL model, and to keep $B_{2}$ to show the general dependence of the phase on the envelope amplitude for noisy limit cycles; the fact that $B_{2}=0$ is just a specific 

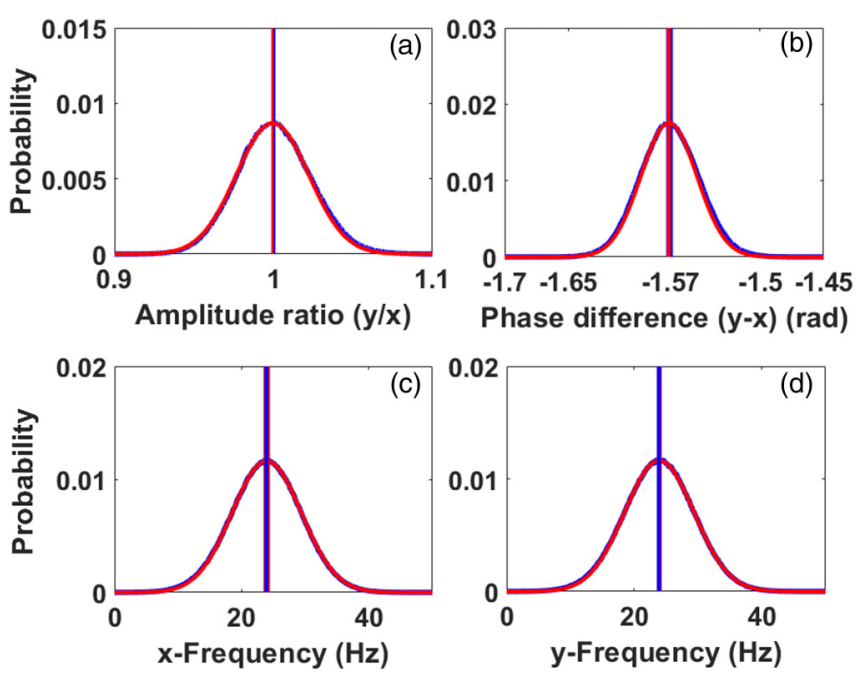

FIG. 8. Amplitude ratio, phase difference between $y$ and $x$ fluctuations, along with their frequency, in the noisy limit-cycle regime. As for the SWC3 case, the amplitude ratio is plotted [red curve (a)] along with the corresponding Gaussian distribution [blue curve (a)]. The vertical red line is the result of the linear stability, while the vertical blue line corresponds to the mean of the Gaussian distribution. Similar results hold for (b)-(d). The probability distributions of the fixed ratio and phase difference are Gaussian distributed. The $x$ and $y$ frequencies are also Gaussian distributed with their mean value at $\omega_{0}$. The values of $\omega_{0}$ were converted to be in the beta range. Parameters are as in Fig. 9.

property of the SSL model. We focus on the noisy limit-cycle regime, comparing our amplitude-phase dynamics with the SAM to those extracted using the Hilbert transform. Figure 9 shows good agreement between the two. Note that we have chosen parameters such that the peak frequency is lower than for the SWC3 model, placing it more in the beta range in the context of brain rhythms. Similar analyses can be performed at higher (gamma) or lower frequencies such as in the delta and theta ranges.

Unlike the SWC3 case, here we have independent correlated OU noise inputs to the $\mathrm{x}$ and $\mathrm{y}$ components. We computed the density $P(Z)$ for different correlation times $\tau=\tau_{x}=\tau_{y}$ (varied while keeping the noise intensity fixed for both inputs), and this was repeated for four noise intensities. Figure 10 shows that shorter noise correlation time and larger intensity, and especially their combination, cause significant probability at small amplitudes, which is a sign of bursting. For small $\tau$, the inputs approach white noise, and the behavior of the amplitude can be understood from the analysis of the SWC3 above. We also observed a good match between the SAM theory and numerical simulations for all correlation times.

As for the SWC3 model, we computed the peak of the probability density of the amplitude process for different $\tau$ values at a low and a high noise strength (Fig. 11). Like previously observed, the noise and correlation strength increase the overall amplitude mean as they smoothe out (i.e., linearize) the supercritical Hopf bifurcation. The SAM approximation continues to work well even for the stronger correlation case.

Next we compare our amplitude-phase decomposition with previous decomposition of the SSL model using the change of variable $\theta(t)=\omega_{0} t+\phi(t)$, leading to

$$
d \theta(t)=\omega_{0} d t+\frac{\sqrt{D}}{Z(t)} d W_{2}(t) .
$$

In the deterministic limit $D=0$, Eqs. (42) and (44) correspond to the polar normal form of the supercritical Hopf bifurcation [78]. In the stochastic case however, the noise appears in a nontrivial manner in both the $Z$ and $\phi$ (or $\theta$ ) equations and is a general amplitude-phase description of noisy oscillators around a supercritical Hopf bifurcation. By taking into account the amplitude ratio and phase difference between the two components, it provides a good description of the quasiellipsoidal form of the quasicycle or of the stochastic
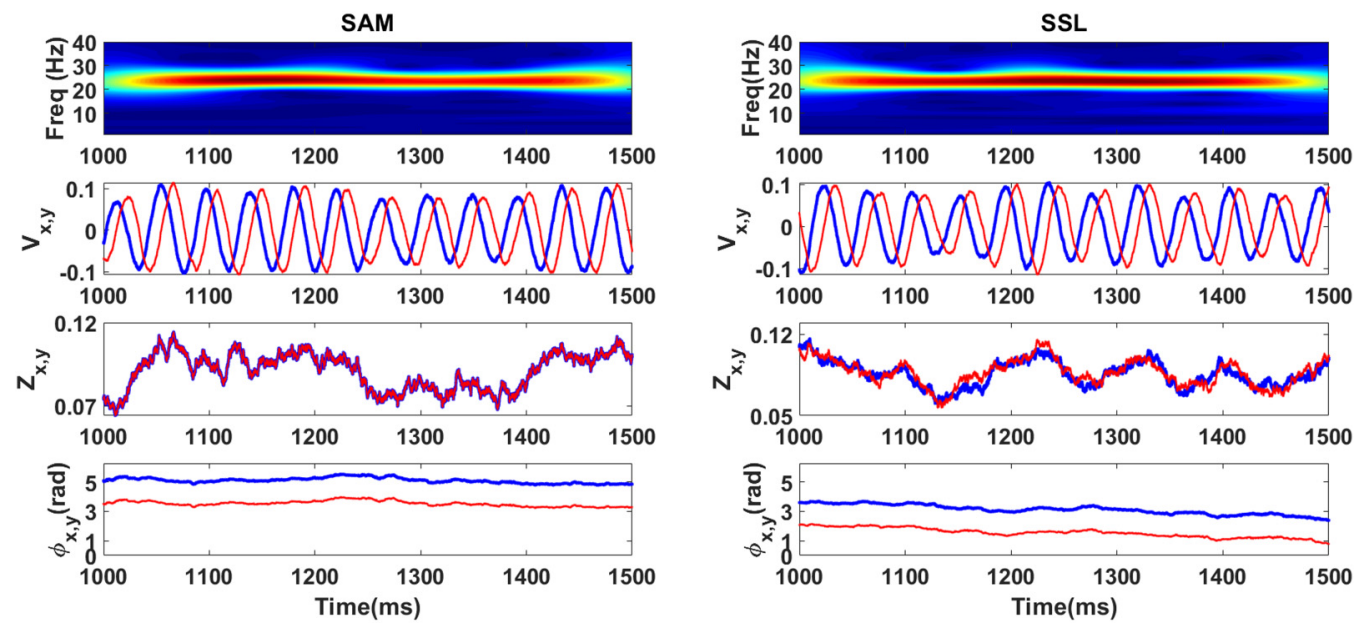

FIG. 9. Spectrogram, $x$ and $y$ fluctuations, amplitudes and phases computed from the SAM and from the SSL using the Hilbert transform. From top to bottom: spectrogram, $x$ and $y$ fluctuations, amplitudes and phases of the $x$ (blue) and $y$ (red) populations computed from the SAM analysis (left) and from the Hilbert transform (right) performed on numerical solutions of the SSL model. The SAM results match well with the numerical results obtained through the Hilbert transform. Parameters are $a_{0}=0.01, \omega_{0}=0.150$, and $\sigma_{x}=\sigma_{y}=\sigma=0.002$. We have consider a Gaussian white noises applied on $x$ and $y$ variables. 

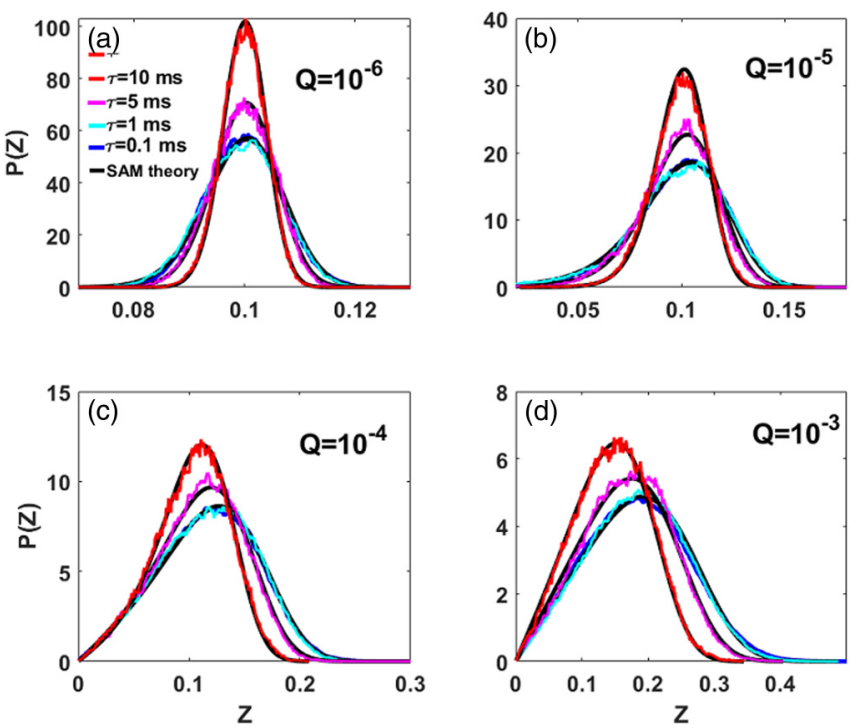

FIG. 10. Effect of noise correlation time and intensity on the SSL amplitude-phase dynamics. The probability densities of the amplitudes of the SSL for correlation times $\tau=\tau_{x}=\tau_{y}$ of the OU processes were computed for (a) weak noise, [(b) and (c)] intermediate noise, and (d) strong noise intensity. We observe that for all intensities and correlation times, the density for the amplitude processes computed numerically through the Hilbert transform (colored curves) match with those calculated theoretically (black curves) using Eqs. (31) and (26), with $\sigma_{E}=\sigma_{x}=\sigma ; \sigma_{I}=\sigma_{y}=\sigma$ and $Q=$ $\tau \sigma^{2}$. For weak values of $\tau$ and large intensities, the corresponding densities are broader. For large intensities, the densities have higher peak amplitude; weak values of $\tau$ also increase the peak amplitude. Parameters are as in Fig. 8 unless otherwise specified here.
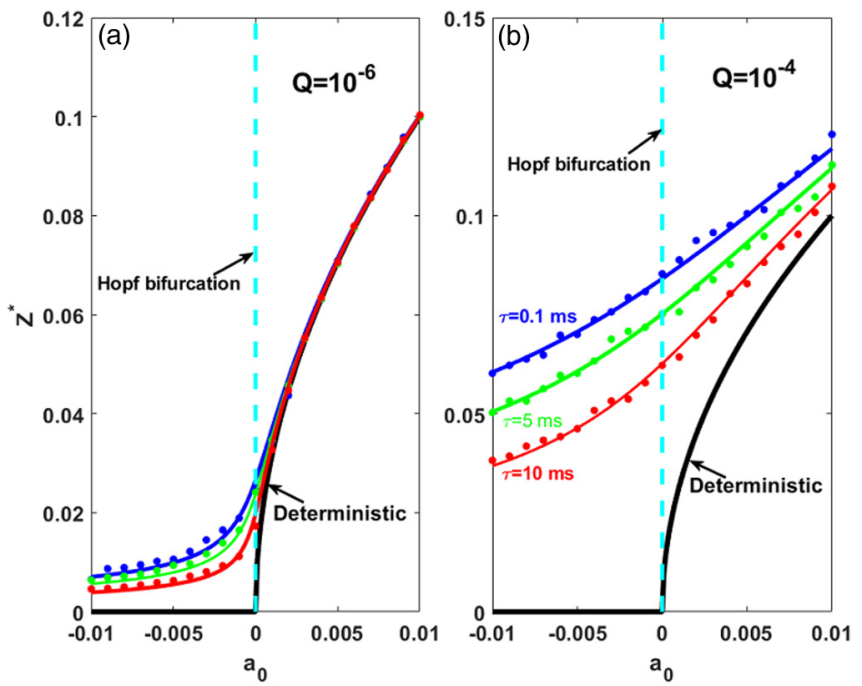

FIG. 11. Bifurcation diagram of the SSL model. The peak of the amplitude probability density is plotted as a function of the bifurcation parameter $a_{0}$. The noise is the OU process with different $\tau=\tau_{x}=\tau_{y}$. The noise strength is (a) $Q=\tau \sigma^{2}=10^{-6}$ and (b) $10^{-4}$. Increasing $Q$ and $\tau$ increases the peak amplitude value. Solid curves correspond to SAM theory while dots are from numerical simulations. For all cases, a good match between theory and numerical results is seen. Parameters are as in Fig. 8 unless otherwise specified. limit cycle (the deterministic limit cycle is a quasiellipsoid for the WC model); it complements other averaging approaches that consider the component amplitudes to be the same $[80,83]$.

\section{AMPLITUDE-PHASE DECOMPOSITION FOR NETWORKS}

Finally we extend our theory to interconnected networks of $E-I$ units described by the SWC3 or SSL nonlinear rate models. One application of such amplitude-phase decomposition may be the study of the functional connectivity between brain areas $[75,76,80]$. We only consider the case where the network frequencies belong to the same band and therefore avoid the case of cross-frequency coupling where faster rhythms are coupled with slower ones [84].

\section{A. Network of stochastic Wilson-Cowan units}

We consider a network of excitatorily delay-coupled SWC3 units:

$$
\begin{gathered}
\frac{d E_{k}(t)}{d t}=-\alpha_{E} E_{k}+\left(1-E_{k}\right) \beta_{E} f\left(s_{E_{k}}\right)+\eta_{E_{k}}, \\
\frac{d I_{k}(t)}{d t}=-\alpha_{I} I_{k}+\left(1-I_{k}\right) \beta_{I} f\left(s_{I_{k}}\right)+\eta_{I_{k}} .
\end{gathered}
$$

The total excitatory $s_{E_{k}}(t)$ and inhibitory $s_{I_{k}}(t)$ synaptic inputs to a neuron population are given by

$$
\begin{aligned}
& s_{E_{k}}(t)=W_{E E}^{k} E_{k}-W_{E I}^{k} I_{k}+h_{E}^{k}+\sum_{l=1, l \neq k}^{N} S_{E E}^{k l} E_{l}\left(t-\tau_{k l}\right), \\
& s_{I_{k}}(t)=W_{I E}^{k} E_{k}-W_{I I}^{k} I_{k}+h_{I}^{k}+\sum_{l=1, l \neq k}^{N} S_{I E}^{k l} E_{l}\left(t-\tau_{k l}\right) .
\end{aligned}
$$

The coefficient $S_{E E}^{k l}$ denotes the long-range excitatory weight from excitatory population $l$ to excitatory population $k$, while $S_{I E}^{k l}$ accounts for the weight from excitatory population $l$ to inhibitory population $k$. As for the case of the single $E-I$ units, we look at the fluctuations of each unit as follows:

$$
E_{k}(t)=E_{0}^{k}+V_{E_{k}}(t) \quad \text { and } \quad I_{k}(t)=I_{0}^{k}+V_{I_{k}}(t)
$$

Each basic $E$ - $I$ units behaves as above, but for simplicity, we keep only the linear coupling terms because we consider weak coupling coefficients $S_{E E}^{k l}$ and $S_{I E}^{k l}$. In this limit, keeping only linear terms is a reasonable approximation since nonlinear terms will be small and contribute little compared to the linear terms. Also, this simplifies the analytical treatment. Therefore, the only difference is the fact that the coefficients of the single unit analysis now have the index $k$ which characterizes the heterogeneity of each network. However, the form of the coefficients of the deviations dynamics are similar. Specifically, the fixed points $\left(E_{0}^{k}, I_{0}^{k}\right)$ now depend on the coupling terms. The fluctuation dynamics are given by the 
$2 N$-dimensional system:

$$
\begin{aligned}
\frac{d V_{E_{k}}}{d t}= & A_{E E}^{k} V_{E_{k}}+A_{E I}^{k} V_{I_{k}}+L_{E E}^{k} V_{E_{k}}^{2} \\
& +L_{E I}^{k} V_{E_{k}} V_{I_{k}}+M_{E I}^{k} V_{I_{k}}^{2}+B_{1 E}^{k} V_{E_{k}}^{3} \\
& +B_{2 E}^{k} V_{E_{k}} V_{I_{k}}^{2}+B_{3 E}^{k} V_{I_{k}} V_{E_{k}}^{2}+B_{4 E}^{k} V_{I_{k}}^{3} \\
& +\sum_{l=1, l \neq k}^{N} C_{E E}^{k l} V_{E_{l}}\left(t-\tau_{k l}\right)+\eta_{E_{k}}, \\
\frac{d V_{I_{k}}}{d t}= & A_{I E}^{k} V_{E_{k}}+A_{I I}^{k} V_{I_{k}}+L_{I I}^{k} V_{I_{k}}^{2} \\
& +L_{I E}^{k} V_{E_{k}} V_{I_{k}}+M_{I E}^{k} V_{E_{k}}^{2}+B_{1 I}^{k} V_{I_{k}}^{3} \\
& +B_{2 I}^{k} V_{E_{k}} V_{I_{k}}^{2}+B_{3 I}^{k} V_{I_{k}} V_{E_{k}}^{2}+B_{4 I}^{k} V_{E_{k}}^{3} \\
& +\sum_{l=1, l \neq k}^{N} C_{I E}^{k l} V_{E_{l}}\left(t-\tau_{k l}\right)+\eta_{I_{k}} .
\end{aligned}
$$

As for the case of a single network we have the following $B_{4 E}^{k}=B_{4 I}^{k}=0$ and $M_{I E}^{k}=M_{E I}^{k}=0$ (see Appendix A Fig. 14). The effective coupling coefficients $C_{E E}^{k l}$ and $C_{E E}^{k l}$ are

$$
\begin{aligned}
C_{E E}^{k l} & =\left(1-E_{0}^{k}\right) \beta_{E} f^{\prime}\left(s_{E_{0}^{k}}\right) S_{E E}^{k l}, \\
C_{I E}^{k l} & =\left(1-I_{0}^{k}\right) \beta_{I} f^{\prime}\left(s_{I_{0}^{k}}\right) S_{I E}^{k l}
\end{aligned}
$$

with the following expressions for the inputs to each population of the $k$ th unit:

$$
\begin{gathered}
s_{E_{0}^{k}}=W_{E E}^{k} E_{0}^{k}-W_{E I}^{k} I_{0}^{k}+h_{E}^{k}+\sum_{l=1, l \neq k}^{N} S_{E E}^{k l} E_{0}^{l}, \\
s_{I_{0}^{k}}=W_{I E}^{k} E_{0}^{k}-W_{I I}^{k} I_{0}^{k}+h_{I}^{k}+\sum_{l=1, l \neq k}^{N} S_{I E}^{k l} E_{0}^{l} .
\end{gathered}
$$

Here $\left(E_{0}^{k}, I_{0}^{k}\right), k=1, \ldots, N$ are the fixed points of the stationary noise-free Eqs. (45) and (46). The coefficients of Eqs. (50-51) have the same form as in the single unit case; however the expressions for $\left(s_{E_{0}}, s_{I_{0}}\right)$ are now replaced by $\left(s_{E_{0}^{k}}, s_{I_{0}^{k}}, k=1, \ldots, N\right)$. Similarly, $\left(E_{0}, I_{0}\right)$ is replaced by $\left(E_{0}^{k}, I_{0}^{k}, k=1, \ldots, N\right)$.

As in the case of the single unit, linear stability analysis suggests a fixed amplitude ratio and phase difference between the $E$ and $I$ components of each unit. However, the determination of these quantities is different from the single unit case. To find the frequency, amplitude ratio and phase difference, these steps are followed.
(1) From the linear stability analysis, compute the eigenvalues of the corresponding $2 \mathrm{~N}$-dimensional linear system associated with Eqs. (50) and (51). The number of eigenvalues is infinite when the propagation delay is considered, and is equal to $2 N$ otherwise. The imaginary part $\omega_{0}$ of the eigenvalue with the largest real part is considered as the principal frequency of the system.

(2) Consider the eigenvalue with the largest real part $(\lambda=$ $\left.v_{\max }+j \omega_{0}\right)$ and solve the following $N \times N$ system of equations for the amplitude ratios derived from the linear stability analysis:

$$
\begin{aligned}
A_{E I}^{k} \tilde{\alpha}_{k k}+\sum_{l=1, l \neq k}^{N} C_{E E}^{k l} \exp \left(-\lambda \tau_{k l}\right) \tilde{\alpha}_{k l} & =\lambda-A_{E E}^{k} \\
\sum_{l=1, l \neq k}^{N} C_{I E}^{k l} \exp \left(-\lambda \tau_{k l}\right) \tilde{\alpha}_{k l}+\left(A_{I I}^{k}-\lambda\right) \tilde{\alpha}_{k k} & =-A_{I E}^{k} \\
\tilde{\alpha}_{k l}=\left(\tilde{\alpha}_{l k}\right)^{-1}, \quad k, \neq l & =1, \ldots, N,
\end{aligned}
$$

where

$$
\tilde{\alpha}_{k k}=\frac{\tilde{B}_{I_{k}}}{\tilde{B}_{E_{k}}}, \quad \tilde{\alpha}_{k l}=\frac{\tilde{B}_{E_{l}}}{\tilde{B}_{E_{k}}}, \quad k, \neq l=1, \ldots, N .
$$

(3) The amplitude ratio and phase difference between inhibitory and excitatory fluctuations are therefore given by

$$
\alpha_{k}=\left|\tilde{\alpha}_{k k}\right| ; \quad \delta_{k}=\operatorname{Arg}\left(\tilde{\alpha}_{k k}\right), \quad k=1, \ldots, N,
$$

where $\|$ and Arg are respectively the modulus and the argument of a complex number.

As in the case of single unit, we can look for solutions of excitatory and inhibitory fluctuation dynamics for each population in each unit in the form:

$$
\begin{aligned}
& V_{E_{k}}(t)=Z_{k}(t) \cos \left(\omega_{0} t+\phi_{k}(t)\right), \\
& V_{I_{k}}(t)=\alpha_{k} Z_{k}(t) \cos \left(\omega_{0} t+\phi_{k}(t)+\delta_{k}\right) .
\end{aligned}
$$

We have dropped the subscript $E$ for the variables $Z$ and $\phi$ for the sake of readability. We then plugged these expressions in the system of $2 N$-dimensional Eqs. (50) and (51) and obtained the final system of $2 N$-dimensional equations for the amplitudes $Z_{k}$ and phases $\phi_{k}$ :

$$
\begin{aligned}
& \frac{d Z_{k}}{d t}=F_{1}^{k}\left(Z_{k}, \phi_{k}\right)+G_{1}^{k}\left(Z_{k}, \phi_{k}, \eta_{E_{k}}, \eta_{I_{k}}\right), \\
& \frac{d \phi_{k}}{d t}=F_{2}^{k}\left(Z_{k}, \phi_{k}\right)+G_{2}^{k}\left(Z_{k}, \phi_{k}, \eta_{E_{k}}, \eta_{I_{k}}\right)
\end{aligned}
$$

with the following functions:

$$
\begin{aligned}
F_{1}^{k}\left(Z_{k}, \phi_{k}\right) & =\frac{1}{\alpha_{k} \sin \left(\delta_{k}\right)}\left[\alpha_{k} f_{1}^{k}\left(Z_{k}, \phi_{k}\right) \sin \left(\omega_{0} t+\phi_{k}+\delta_{k}\right)-f_{2}^{k}\left(Z_{k}, \phi_{k}\right) \sin \left(\omega_{0} t+\phi_{k}\right)\right], \\
F_{2}^{k}\left(Z_{k}, \phi_{k}\right) & =\frac{1}{\alpha_{k} Z_{k} \sin \left(\delta_{k}\right)}\left[\alpha_{k} f_{1}^{k}\left(Z_{k}, \phi_{k}\right) \cos \left(\omega_{0} t+\phi_{k}+\delta_{k}\right)-f_{2}^{k}\left(Z_{k}, \phi_{k}\right) \cos \left(\omega_{0} t+\phi_{k}\right)\right], \\
G_{1}^{k}\left(Z_{k}, \phi_{k}, \eta_{E_{k}}, \eta_{I_{k}}\right) & =\frac{1}{\alpha_{k} \sin \left(\delta_{k}\right)}\left[\alpha_{k} g_{1}^{k}\left(Z_{k}, \phi_{k}, \eta_{E_{k}}, \eta_{I_{k}}\right) \sin \left(\omega_{0} t+\phi_{k}+\delta_{k}\right)-g_{2}^{k}\left(Z_{k}, \phi_{k}, \eta_{E_{k}}, \eta_{I_{k}}\right) \sin \left(\omega_{0} t+\phi_{k}\right)\right], \\
G_{2}^{k}\left(Z_{k}, \phi_{k}, \eta_{E_{k}}, \eta_{I_{k}}\right) & =\frac{1}{\alpha_{k} Z_{k} \sin \left(\delta_{k}\right)}\left[\alpha_{k} g_{1}^{k}\left(Z_{k}, \phi_{k}, \eta_{E_{k}}, \eta_{I_{k}}\right) \cos \left(\omega_{0} t+\phi_{k}+\delta_{k}\right)-g_{2}^{k}\left(Z_{k}, \phi_{k}, \eta_{E_{k}}, \eta_{I_{k}}\right) \cos \left(\omega_{0} t+\phi_{k}\right)\right],
\end{aligned}
$$




$$
\begin{aligned}
f_{1}^{k}\left(Z_{k}, \phi_{k}\right)= & \omega_{0} Z_{k} \sin \left(\omega_{0} t+\phi_{k}\right)+A_{E E}^{k} Z_{k} \cos \left(\omega_{0} t+\phi_{k}\right)+A_{E I}^{k} \alpha_{k} Z_{k} \cos \left(\omega_{0} t+\phi_{k}+\delta_{k}\right) \\
& +L_{E E}^{k} Z_{k}^{2} \cos ^{2}\left(\omega_{0} t+\phi_{k}\right)+L_{E I}^{k} \alpha_{k} Z_{k}^{2} \cos \left(\omega_{0} t+\phi_{k}\right) \cos \left(\omega_{0} t+\phi_{k}+\delta_{k}\right) \\
& +B_{1 E}^{k} Z_{k}^{3} \cos ^{3}\left(\omega_{0} t+\phi_{k}\right)+B_{2 E}^{k} \alpha_{k}^{2} Z_{k}^{3} \cos \left(\omega_{0} t+\phi_{k}\right) \cos ^{2}\left(\omega_{0} t+\phi_{k}+\delta_{k}\right) \\
& +B_{3 E}^{k} \alpha_{k} Z_{k}^{3} \cos ^{2}\left(\omega_{0} t+\phi_{k}\right) \cos \left(\omega_{0} t+\phi_{k}+\delta_{k}\right)+\sum_{l=1, l \neq k}^{N} C_{E E}^{k l} Z_{l}\left(t-\tau_{k l}\right) \cos \left[\omega_{0} t-\omega_{0} \tau_{k l}+\phi_{l}\left(t-\tau_{k l}\right)\right], \\
f_{2}^{k}\left(Z_{k}, \phi_{k}\right)= & \alpha_{k} \omega_{0} Z_{k} \sin \left(\omega_{0} t+\phi_{k}+\delta_{k}\right)+A_{I E}^{k} Z_{k} \cos \left(\omega_{0} t+\phi_{k}\right)+A_{I I}^{k} \alpha_{k} Z_{k} \cos \left(\omega_{0} t+\phi_{k}+\delta_{k}\right) \\
& +L_{I I}^{k} \alpha_{k}^{2} Z_{k}^{2} \cos ^{2}\left(\omega_{0} t+\phi_{k}+\delta_{k}\right)+L_{I E}^{k} \alpha_{k} Z_{k}^{2} \cos \left(\omega_{0} t+\phi_{k}\right) \cos \left(\omega_{0} t+\phi_{k}+\delta_{k}\right) \\
& +B_{1 I}^{k} \alpha_{k}^{3} Z_{k}^{3} \cos ^{3}\left(\omega_{0} t+\phi_{k}+\delta_{k}\right)+B_{2 I}^{k} \alpha_{k}^{2} Z_{k}^{3} \cos \left(\omega_{0} t+\phi_{k}\right) \cos { }^{2}\left(\omega_{0} t+\phi_{k}+\delta_{k}\right) \\
& +B_{3 I}^{k} \alpha_{k} Z_{k}^{3} \cos ^{2}\left(\omega_{0} t+\phi_{k}\right) \cos \left(\omega_{0} t+\phi_{k}+\delta_{k}\right)+\sum_{l=1, l \neq k}^{N} C_{I E}^{k l} Z_{l}\left(t-\tau_{k l}\right) \cos \left[\omega_{0} t-\omega_{0} \tau_{k l}+\phi_{l}\left(t-\tau_{k l}\right)\right],
\end{aligned}
$$$$
g_{1}^{k}\left(Z_{k}, \phi_{k}, \xi_{E_{k}}, \xi_{I_{k}}\right)=\eta_{E_{k}}(t) ; \quad g_{2}^{k}\left(Z_{k}, \phi_{k}, \xi_{E_{k}}, \xi_{I_{k}}\right)=\eta_{I_{k}}(t) \text {. }
$$

Applying the SAM method to the $2 N$-dimensional system [Eqs. (55) and (56)] leads to the following amplitude-phase equations for the networks of SWC3 units:

$$
\begin{gathered}
d Z_{k}(t)=\left\{-\lambda_{k} Z_{k}(t)+B_{1}^{k} Z_{k}^{3}(t)+\frac{D_{k}}{2 Z_{k}(t)}+\frac{1}{2 \alpha_{k} \sin \left(\delta_{k}\right)} \sum_{l=1, l \neq k}^{N}\left[\alpha_{k} C_{E E}^{k l} \sin \left(\phi_{k}(t)-\phi_{l}\left(t-\tau_{k l}\right)+\omega_{0} \tau_{k l}+\delta_{k}\right)\right.\right. \\
\left.\left.-C_{I E}^{k l} \sin \left(\phi_{k}(t)-\phi_{l}\left(t-\tau_{k l}\right)+\omega_{0} \tau_{k l}\right)\right] Z_{l}\left(t-\tau_{k l}\right)\right\} d t+\sqrt{D_{k}} d W_{1}^{k}(t), \\
d \phi_{k}(t)=\left\{\Omega_{k}+B_{2}^{k} Z_{k}^{2}(t)+\frac{1}{2 \alpha_{k} \sin \left(\delta_{k}\right)} \sum_{l=1, l \neq k}^{N}\left[\alpha_{k} C_{E E}^{k l} \cos \left(\phi_{k}(t)-\phi_{l}\left(t-\tau_{k l}\right)+\omega_{0} \tau_{k l}+\delta_{k}\right)\right.\right. \\
\left.\left.-C_{I E}^{k l} \cos \left(\phi_{k}(t)-\phi_{l}\left(t-\tau_{k l}\right)+\omega_{0} \tau_{k l}\right)\right] \frac{Z_{l}\left(t-\tau_{k l}\right)}{Z_{k}(t)}\right\} d t+\frac{\sqrt{D_{k}}}{Z_{k}(t)} d W_{2}^{k}(t) .
\end{gathered}
$$

The coefficients in these expressions are defined by

$$
B_{1}^{k}=\frac{1}{8}\left[3 B_{1 E}^{k}+B_{3 I}^{k}+\alpha_{k}^{2}\left(B_{2 E}^{k}+3 B_{1 I}^{k}\right)+2 \alpha_{k} \cos \left(\delta_{k}\right)\left(B_{3 E}^{k}+B_{2 I}^{k}\right)\right]
$$

as well as

$$
B_{2}^{k}=\frac{1}{8 \sin \left(\delta_{k}\right)}\left[2 \alpha_{k}\left(B_{3 E}^{k}-B_{2 I}^{k}\right)+3\left(B_{1 E}^{k}-B_{3 I}^{k}\right) \cos \left(\delta_{k}\right)+3 \alpha_{k}^{2}\left(B_{2 E}^{k}-B_{1 I}^{k}\right) \cos \left(\delta_{k}\right)+\alpha_{k}\left(B_{3 E}^{k}-B_{2 I}^{k}\right) \cos \left(2 \delta_{k}\right)\right]
$$

with the definitions

$$
\begin{aligned}
\lambda_{k} & =-\frac{A_{E E}^{k}+A_{I I}^{k}}{2}, \quad \Omega_{k}=-\omega_{0}+\frac{\alpha_{k} \cos \left(\delta_{k}\right)\left(A_{E E}^{k}-A_{I I}^{k}\right)+\alpha_{k}^{2} A_{E I}^{k}-A_{I E}^{k}}{2 \alpha_{k} \sin \left(\delta_{k}\right)}, \\
D_{k} & =\frac{1}{2 \alpha_{k}^{2} \sin ^{2}\left(\delta_{k}\right)}\left[\frac{2 \tau_{E_{k}}\left(\alpha_{k} \sigma_{E_{k}}\right)^{2}}{1+\left(\omega_{0} \tau_{E_{k}}\right)^{2}}+\frac{2 \tau_{I_{k}}\left(\sigma_{I_{k}}\right)^{2}}{1+\left(\omega_{0} \tau_{I_{k}}\right)^{2}}\right]=\frac{\pi}{\alpha_{k}^{2} \sin ^{2}\left(\delta_{k}\right)}\left[\alpha_{k}^{2} S_{\xi_{E_{k}}}\left(\omega_{0}\right)+S_{\xi_{l_{k}}}\left(\omega_{0}\right)\right] .
\end{aligned}
$$

We have thus derived an amplitude-phase decomposition of connected SWC3 E-I units through the SAM by extending the technique used for an isolated $E-I$ network. The result is a set of $2 \times N$ stochastic differential equations for the amplitudes and phases of each unit. The connectivity between the amplitude and phase are made through sinusoidal coupling functions. Unfortunately, the amplitude equation is not uncoupled from that for the phase as was the case for a single unit. A Fokker-Planck analysis is therefore difficult to conduct.

However, we performed numerical simulations of this network of amplitude-phase dynamics [Eqs. (57) and (58)] and their original fluctuations variables [Eqs. (50) and (51)] and found good agreement between the two. This was done for the quasicycle regimes as well as for the noisy limit-cycle regime in Figs. 12(a) and 12(b). For each regime, we made sure that a single isolated $E-I$ unit was in the regime of interest (quasi- or limit cycle), and that the connectivity between units kept the network in the same regime of each isolated unit. In other words, the connectivity does not induce a change of regime.

We represented the probability densities of the amplitude $Z_{k}, k=1,2,3$ for three selected units in a minimal heterogeneous network of 5 units and the corresponding excitatory amplitudes extracted using the Hilbert transform for Eqs. (50) and (51) and found good agreement between the two methods. In Figs. 12(a) and 12(b), the black curves are the result of the SAM approximation, while the colored curves correspond to the Hilbert transform computation. Our amplitude-phase approach across 
the Hopf bifurcation is therefore generally applicable to both single units and heterogeneous networks of units in both the quasiand limit-cycle regimes.

To recover the common fast phase variable usually used and associated to the Hilbert transform, it suffices to consider the change of variable $\theta_{k}(t)=\omega_{0} t+\phi_{k}(t)$. This leads to the following amplitude-phase dynamics

$$
\begin{gathered}
d Z_{k}(t)=\left\{-\lambda_{k} Z_{k}(t)+B_{1}^{k} Z_{k}^{3}(t)+\frac{D_{k}}{2 Z_{k}(t)}+\frac{1}{2 \alpha_{k} \sin \left(\delta_{k}\right)} \sum_{l=1, l \neq k}^{N}\left[\alpha_{k} C_{E E}^{k l} \sin \left(\theta_{k}(t)-\theta_{l}\left(t-\tau_{k l}\right)+\delta_{k}\right)\right.\right. \\
\left.\left.-C_{I E}^{k l} \sin \left(\theta_{k}(t)-\theta_{l}\left(t-\tau_{k l}\right)\right)\right] Z_{l}\left(t-\tau_{k l}\right)\right\} d t+\sqrt{D_{k}} d W_{1}^{k}(t) \\
d \theta_{k}(t)=\left\{\omega_{k}+B_{2}^{k} Z_{k}^{2}(t)+\frac{1}{2 \alpha_{k} \sin \left(\delta_{k}\right)} \sum_{l=1, l \neq k}^{N}\left[\alpha_{k} C_{E E}^{k l} \cos \left(\theta_{k}(t)-\theta_{l}\left(t-\tau_{k l}\right)+\delta_{k}\right)\right.\right. \\
\left.\left.-C_{I E}^{k l} \cos \left(\theta_{k}(t)-\theta_{l}\left(t-\tau_{k l}\right)\right)\right] \frac{Z_{l}\left(t-\tau_{k l}\right)}{Z_{k}(t)}\right\} d t+\frac{\sqrt{D_{k}}}{Z_{k}(t)} d W_{2}^{k}(t)
\end{gathered}
$$

The new parameter $\omega_{k}$ is related to $\Omega_{k}$ and depends on the intrinsic parameter of each network, as following:

$$
\begin{aligned}
\omega_{k} & =\Omega_{k}+\omega_{0} \\
& =\frac{\alpha_{k} \cos \left(\delta_{k}\right)\left(A_{E E}^{k}-A_{I I}^{k}\right)+\alpha_{k}^{2} A_{E I}^{k}-A_{I E}^{k}}{2 \alpha_{k} \sin \left(\delta_{k}\right)} .
\end{aligned}
$$

It is related to the deterministic frequency of the oscillator $k$ and coincides with the imaginary part of the complex con-
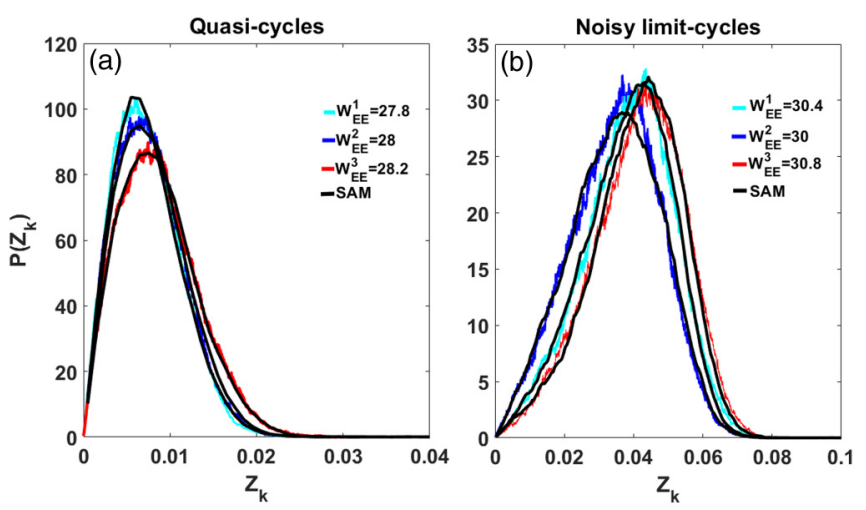

FIG. 12. Envelope probability densities of 3 selected units in a heterogeneous network of 5 SWC3 units. Numerical simulations of the SWC3 network of units [Eqs. (50) and (51)] followed by Hilbert transforms to obtain the amplitude density, along with numerical simulations of the corresponding amplitude-phase decomposition obtained through the SAM [Eqs. (57) and (58)]. (a) quasicycle regime. Each unit is in this regime when isolated or coupled. Parameters are $W_{E E}^{k}=27.8,28,28.2,27.6,28.4, k=1, \ldots, 5$. The network is all-to-all connected with $C_{I E}^{k l}=C_{I E}=0, \tau_{k l}=0$, and $C_{E E}^{k l}=C_{E E}=2 / 5$. (b) Noisy limit-cycle regime. Each isolated network is in the limit-cycle regime and remains so when connected. Parameters are $W_{E E}^{k}=30.4,30,30.8,30.6,30.4, k=1, \ldots, 5$. The connectivity is all-to-all with $C_{I E}^{k l}=C_{I E}=0, \tau_{k l}=0$ and $C_{E E}^{k l}=$ $C_{E E}=1 / 5$. Black curves correspond to the SAM approximation Eqs. (57) and (58); colored curves correspond to the approximate SWC3. An excellent match is seen between the amplitude densities computed from the SAM and the original model for both regimes. Noise parameters for each unit are: $\sigma_{E}=0.0014, \sigma_{I}=$ 0.0048 , and $\sigma=0.005$. Other parameters are specified in Fig. 1 . jugate eigenvalues $\omega_{0}$ for a single oscillator. However, we found that the complete deterministic frequency is generally amplitude-dependent as shown by the two first terms in right side of the equality of Eq. (62). The news dynamics Eqs. (61) and (62) represent a general model for the interaction of
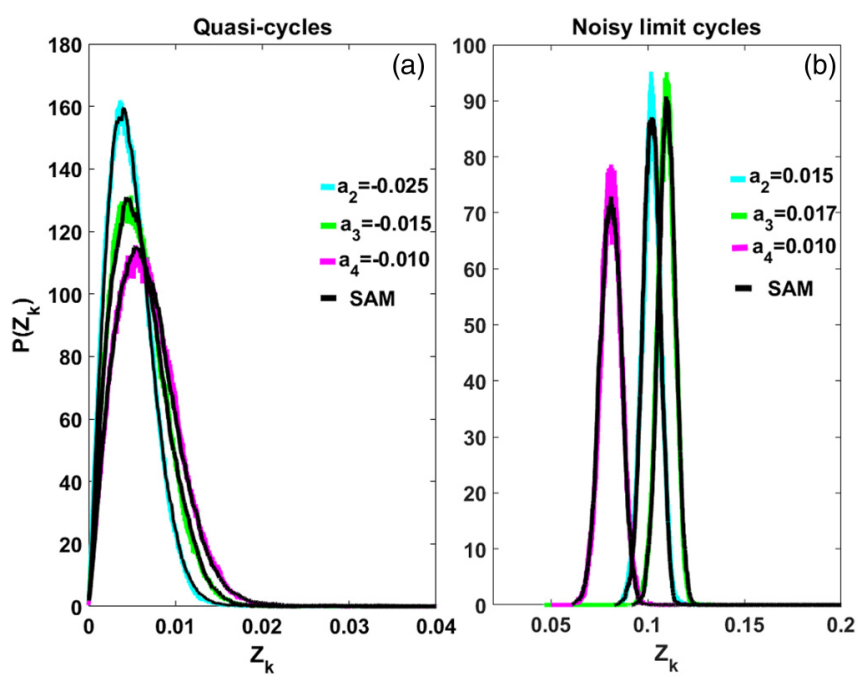

FIG. 13. Amplitude probability densities of 3 selected units in a network of 5 SSL units. Numerical simulations of the amplitude equations obtained from the SAM Eqs. (66) and (67) and of the Hilbert transform extracted from simulations of the SSL model. (a) Quasicycle regime. Each unit is in this regime when isolated or coupled. Parameters are $a_{k}=-0.01,-0.025,-0.02,-0.01$, -0.01 , and $k=1, \ldots, 5$. The network is all-to-all connected with $C_{k l}=K / N=0.01 / 5$ and $\tau_{k l}=0$. (b) Noisy limit-cycle regime. Each isolated or coupled network is in the limit-cycle regime. Parameters are $a_{k}=0.015,0.017,0.01,0.01,0.01$, and $k=1, \ldots, 5$. The connectivity is all-to-all with $C_{k l}=K / N=0.01 / 5$ and $\tau_{k l}=0$. Black curves correspond to the SAM Eqs. (66) and (67) and colored curves to the original SSL dynamics. An excellent match is again seen between the two envelope dynamics in both regimes. Noise parameters for each unit are: $\sigma_{x}=\sigma_{y}=\sigma=0.001$ and the parameter $b_{0}$ is set to 0.15 . For the two regimes, the coupling coefficient $C_{k l}$ is applied only on the $x$ variable for simplicity. Other parameters are specified in Fig. 9. 
oscillators in the slow $Z_{k}(t)$ and fast $\theta_{k}(t)$ variables framework. The major difference with the previous amplitude-phase dynamics Eqs. (57) and (58) is the timescale of the new phase variable $\theta_{k}(t)$ which now evolves on a faster time scale compared to the amplitude $Z_{k}(t)$ which remain in a slow timescale. This new framework is usually adopted when defining the interaction of oscillators in the amplitude-phase representation.

\section{B. Networks of stochastic Stuart-Landau units}

\section{General case}

We now consider a network of coupled SSL units $[85,86]$. The coupling is a linear coupling, the model is described as

$$
\begin{aligned}
& \dot{x}_{k}(t)=a_{k} x_{k}(t)-\omega_{0}^{k} y_{k}(t)-x_{k}(t)\left[x_{k}^{2}(t)+y_{k}^{2}(t)\right]+\sum_{l=1, l \neq k}^{N} C_{k l}\left[x_{l}\left(t-\tau_{k l}\right)-x_{k}(t)\right]+\eta_{x}^{k}(t), \\
& \dot{y}_{k}(t)=\omega_{0}^{k} x_{k}(t)+a_{k} y_{k}(t)-y_{k}(t)\left[x_{k}^{2}(t)+y_{k}^{2}(t)\right]+\sum_{l=1, l \neq k}^{N} C_{k l}\left[y_{l}\left(t-\tau_{k l}\right)-y_{k}(t)\right]+\eta_{y}^{k}(t) .
\end{aligned}
$$

We perform the same analysis as for the case of the network of SWC3 units. The fluctuations from the fixed points are therefore sought in the form

$$
V_{x}^{k}(t)=Z_{k}(t) \cos \left[\omega_{0} t+\phi_{k}(t)\right], \quad V_{y}^{k}(t)=\alpha_{k} Z_{k}(t) \cos \left[\omega_{0} t+\phi_{k}(t)+\delta_{k}\right] .
$$

The same steps as for the SWC3 units lead to amplitude-phase equations for the network of coupled SSL units:

$$
\begin{aligned}
d Z_{k}(t)= & \left\{-\lambda_{k} Z_{k}(t)+B_{1}^{k} Z_{k}^{3}(t)+\frac{D_{k}}{2 Z_{k}(t)}+\frac{1}{2 \alpha_{k} \sin \left(\delta_{k}\right)} \sum_{l=1, l \neq k}^{N} C_{k l}\left[\alpha _ { k } \left(Z_{l}\left(t-\tau_{k l}\right) \sin \left(\phi_{k}(t)-\phi_{l}\left(t-\tau_{k l}\right)+\omega_{0} \tau_{k l}+\delta_{k}\right)\right.\right.\right. \\
- & \left.\left.\left.Z_{k}(t) \sin \left(\delta_{k}\right)\right)+\left(\alpha_{l} Z_{l}\left(t-\tau_{k l}\right) \sin \left(\phi_{l}\left(t-\tau_{k l}\right)-\omega_{0} \tau_{k l}-\phi_{k}(t)+\delta_{l}\right)-\alpha_{k} Z_{k}(t) \sin \left(\delta_{k}\right)\right)\right]\right\} d t+\sqrt{D_{k}} d W_{1}^{k}(t), \\
d \phi_{k}(t)= & \left\{\Omega_{k}+B_{2}^{k} Z_{k}^{2}(t)+\frac{1}{2 \alpha_{k} \sin \left(\delta_{k}\right)} \sum_{l=1, l \neq k}^{N} C_{k l}\left[\alpha_{k} \cos \left(\phi_{k}(t)-\phi_{l}\left(t-\tau_{k l}\right)+\omega_{0} \tau_{k l}+\delta_{k}\right)\right.\right. \\
& \left.\left.-\alpha_{l} \cos \left(\phi_{l}\left(t-\tau_{k l}\right)-\omega_{0} \tau_{k l}-\phi_{k}(t)+\delta_{l}\right)\right] \frac{Z_{l}\left(t-\tau_{k l}\right)}{Z_{k}(t)}\right\} d t+\frac{\sqrt{D_{k}}}{Z_{k}(t)} d W_{2}^{k}(t) .
\end{aligned}
$$

The amplitude equations are now coupled with the phase equations, making it now difficult to perform a Fokker-Planck analysis in contrast to the case of the isolated unit. Figure 13 shows results from numerical simulations of the amplitude-phase equations for a small all-to-all connected heterogeneous network of five units. Only the parameters $a_{k}$ differ amongst the units. The parameters $a_{k}$ are such that each isolated unit is in the quasicycle regime. The connectivity coefficient is then chosen to ensure that the connected network remains in the quasicycle regime. The same approach is used for the limit-cycle regime. The results of numerical simulations in Fig. 13 show good agreement between the envelopes computed on the original SSL model using the Hilbert transform (colored curves) and those simulated from the SAM dynamics (black curves). Our analysis thus works well for a single unit for a different model than SWC3, for different types of stochastic input, for low and high frequency bands, and for networks of several units. As for the case of the SWC3, we can rewritte the dynamics Eqs. (66) and (67) in the framework of the slow amplitude and fast phase by using the change of variable $\theta_{k}(t)=\omega_{0} t+\phi_{k}(t)$,

$$
\begin{gathered}
d Z_{k}(t)=\left\{-\lambda_{k} Z_{k}(t)+B_{1}^{k} Z_{k}^{3}(t)+\frac{D_{k}}{2 Z_{k}(t)}+\frac{1}{2 \alpha_{k} \sin \left(\delta_{k}\right)} \sum_{l=1, l \neq k}^{N} C_{k l}\left[\alpha _ { k } \left(Z_{l}\left(t-\tau_{k l}\right) \sin \left(\theta_{k}(t)-\theta_{l}\left(t-\tau_{k l}\right)+\delta_{k}\right)\right.\right.\right. \\
\left.\left.\left.-Z_{k}(t) \sin \left(\delta_{k}\right)\right)+\left(\alpha_{l} Z_{l}\left(t-\tau_{k l}\right) \sin \left(\theta_{l}\left(t-\tau_{k l}\right)-\theta_{k}(t)+\delta_{l}\right)-\alpha_{k} Z_{k}(t) \sin \left(\delta_{k}\right)\right)\right]\right\} d t+\sqrt{D_{k}} d W_{1}^{k}(t), \\
d \theta_{k}(t)=\left\{\omega_{k}+B_{2}^{k} Z_{k}^{2}(t)+\frac{1}{2 \alpha_{k} \sin \left(\delta_{k}\right)} \sum_{l=1, l \neq k}^{N} C_{k l}\left[\alpha_{k} \cos \left(\theta_{k}(t)-\theta_{l}\left(t-\tau_{k l}\right)+\delta_{k}\right)\right.\right. \\
\left.\left.-\alpha_{l} \cos \left(\theta_{l}\left(t-\tau_{k l}\right)-\theta_{k}(t)+\delta_{l}\right)\right] \frac{Z_{l}\left(t-\tau_{k l}\right)}{Z_{k}(t)}\right\} d t+\frac{\sqrt{D_{k}}}{Z_{k}(t)} d W_{2}^{k}(t) .
\end{gathered}
$$

\section{Near identical, weakly coupled limit cycles with low noise}

Let us consider the fast phase dynamics of the network of SSL units extracted used the SAM and the change of variable $\theta_{k}(t)=\omega_{0} t+\phi_{k}(t)$ in Eq. (69). If the units are nearly identical, weakly coupled and subject to small noise $\left(D_{k} \ll 1\right)$, we 
can make the following approximations: (1) $\alpha_{k} \sim \alpha_{l} ; \delta_{k} \sim \delta_{l}$ and (2) $Z_{k}(t) \sim Z_{l}(t) \sim Z_{l}\left(t-\tau_{k l}\right) \sim\left\langle Z_{k}(t)\right\rangle=c_{0}$, where $c_{0}$ is a constant. The first approximation comes from the fact that the networks are nearly identical. It reduces the coupling function of the fast phase dynamics Eq. (69) to $C_{k l} \frac{Z_{l}\left(t-\tau_{k l}\right)}{Z_{k}(t)} \sin \left[\theta_{l}\left(t-\tau_{k l}\right)-\theta_{k}(t)\right]$. The second approximation can be made because the units are nearly identical, weakly coupled and the noise is small. The amplitudes then vary slightly over time and we can replace them by a constant. We choose this constant here to be the mean amplitude over the period of a unit and is thus the same for every unit. Such an approximation further reduces the coupling to $C_{k l} \sin \left[\theta_{l}\left(t-\tau_{k l}\right)-\theta_{k}(t)\right]$, and the stochastic terms to $\sqrt{D_{k}^{0}}=\sqrt{D_{k}} / c_{0}$. We recall that for the case of the SL model the coefficients of the squared amplitude in the phase dynamics are always zero $\left(B_{2}^{k}=0\right)$. This leads to the following equation for the phase dynamics:

$$
\frac{d \theta_{k}}{d t}=\omega_{k}+\sum_{l=1, l \neq k}^{N} C_{k l} \sin \left(\theta_{l}\left(t-\tau_{k l}\right)-\theta_{k}(t)\right)+\sqrt{D_{k}^{0}} \xi_{2}^{k},
$$

where

$$
\omega_{k}=\omega_{0}+\Omega_{k}=\frac{\alpha_{k} \cos \left(\delta_{k}\right)\left(A_{x x}^{k}-A_{y y}^{k}\right)+\alpha_{k}^{2} A_{x y}^{k}-A_{y x}^{k}}{2 \alpha_{k} \sin \left(\delta_{k}\right)} .
$$

For the SSL model, it is found that the value of the frequency $\omega_{k}$ coincides with the frequency $\omega_{k}^{0}$ of each isolated unit. Equation (70) is the delayed $[87,88]$ and stochastic [89-92] version of the Kuramoto phase model.

\section{No propagation delay: The Kuramoto model}

If we further neglect the propagation delays $\left(\tau_{k l}=0\right)$ and the noise $D_{k}=0$, we recover the Kuramoto phase model of coupled phase oscillators [93-98]:

$$
\frac{d \theta_{k}(t)}{d t}=\omega_{k}+\sum_{l=1}^{N} C_{k l} \sin \left(\theta_{l}(t)-\theta_{k}(t)\right) .
$$

Note that our amplitude-phase model derived above also displays interesting phase synchronization properties that will be studied elsewhere, since our focus here has been on illustrating the amplitude dynamics. The generality of our model is highlighted by this relation to the well-known deterministic Kuramoto model in specific limits.

\section{DISCUSSION}

In this work, we have addressed the problem of amplitudephase decomposition of noisy oscillations. The quantities of interest are the fluctuations from the fixed points. Our approach consists in first performing a linear stability analysis to extract the amplitude ratio, phase difference and mean frequency. Second, solutions in a sinusoidal form are sought that take into account these properties. And finally the stochastic averaging method (SAM) is applied to obtain the dynamics of the amplitude and phase processes. The method was shown to work well on two different models that span the Hopf bifurcation from the quasicycle to the limit-cycle regimes, as well as for colored noise or Gaussian white noise. It complements previous approaches that treat these two regimes with separate models by providing a unified approach across regimes, and further extends the method to several delay-coupled oscillatory networks.

We first considered a single $E$-I network in the SWC model case, or a single Hopf oscillator in the SSL case. The amplitude dynamics is then uncoupled from the phase dynamics. We then applied a Fokker-Planck analysis to compute the stationary probability densities of the amplitude variables. The peak of the probability densities can be extracted and used as bifurcation parameter. The bifurcation diagram computed with the peak probability density captures the transition from quasi- to limit-cycle oscillations. It shows how noise properties (strength, correlation time) shape the behavior of the oscillations.
Larger noise intensities and shorter noise correlation times increase the peak of the amplitude of the fast oscillation. In the limit-cycle case, the variance of the envelope process also increases, making bursting epochs, i.e., segments where the amplitude process is large - more prominent. This novel result highlights a benefit of considering the amplitude process rather than just the phase when modeling limit-cycle oscillations. The phase dynamics is coupled to the amplitude dynamics. The strength of the noise perturbation on the phase process depends on the amplitude process. Hence during such bursts, the effect of noise weakens [due to the $1 / Z(t)$ dependence]. However, during epochs of low amplitude between bursts, the phase fluctuates more strongly. The amplitude process therefore plays an important role in the phase dynamics, especially for moderate to strong noise.

We extended our method to several coupled units of the stochastic Wilson-Cowan (SWC) and the stochastic StuartLandau (SSL) models. Such super-networks of units have been invoked to model brain connectivity [76,80,99]. Our analysis allowed us to also derive an amplitude-phase model for coupled units. Unlike the single unit network, the amplitude processes are now reciprocally connected with the phase processes of the units. A Fokker-Planck analysis of the system of coupled units is beyond the scope of our study and poses serious challenges; we therefore presented only numerical simulations. However, nevertheless the amplitude-phase dynamics provide a new tool to understand amplitude-phase 
coupling of rhythms that are either autonomous or owe their existence to noise.

As for the case of a single oscillating unit, the SAM method applied to these networks of units of nonlinear oscillators is accurate across the Hopf bifurcation. Future work could investigate the case of a mixture of quasicycle and limit-cycle units and the one where the coupling actually alters the dynamical regime seen in the isolated units. Also, we mostly focused on the dynamics of the amplitude process of an isolated unit since we were mostly interested in the ability of noise in creating bursts. But for the coupled network of oscillators, phase synchronization $[100,101]$ is also of interest. This behavior is present in our coupled models. It is likely that other exciting phenomena such as chimera $[102,103]$ will be revealed in these networks, even in the quasicycle regime.

Note that in the WC framework, the amplitude of the oscillation is a reasonable proxy for the synchronization of an actual network consisting of many noisy excitatory and inhibitory neurons, as established in Ref. [31]. This correspondence will nevertheless miss out on effects such as synchrony without oscillation or strongly synchronous oscillations at low firing rate in deterministic networks [104], a direction we leave for future study.

Although we developed our theory of networks of coupled units with delays using the SAM approach, we have illustrated our results only for zero delays due to space limitations. Recent work shows that interesting phenomena in two coupled quasicycles relate to these delays, such as states of phase locking with phase difference between $-\pi$ and $\pi$ and random changes in the identity of the quasicycle that leads the other [69]. Thus an extension to explore the effects of propagation delays is warranted, which will provide a closer match to the biophysical situation. Our formalism also enables the inclusion of external periodic inputs, which will also be the focus of future work.

Our results point to a possible discrimination between bursting behaviors from two dynamical origins: in quasicycles at low to moderate noise, and in limit cycles at stronger noise. While the spectrograms may be similar, the different shapes of the amplitude density-Rayleigh in the former case and almost Gaussian in the latter-can help distinguish between these two cases. It is possible that further differentiation can be made upon substituting colored noise in the place of Gaussian white noise. The SAM requires the noise to be broadband in order for it to work accurately; Ornstein-Uhlenbeck noise for example would have to be sufficiently broadband. We have also investigated this question over a limited volume of the whole parameter space. It may be easier to discriminate the two kinds of burst dynamics in the neural context of amplitudes of lower frequency rhythms such as in the beta band $(13-30 \mathrm{~Hz})$, in contrast to our focus on the gamma band. Other model topologies than the generic $E-I$ one considered here may also be better suited to such rhythms. The investigation of the amplitude-phase dynamics of such models across the Hopf bifurcation with different types of noises, following the methods described here, is certainly an interesting direction to pursue.

\section{ACKNOWLEDGMENT}

The work was supported by NSERC Canada.

\section{APPENDIX}

\section{Derivation of the SWC3 model}

We consider the Wilson-Cowan equations (1) and (2) in the deterministic limit:

$$
\begin{gathered}
\frac{d E(t)}{d t}=-\alpha_{E} E(t)+(1-E(t)) \beta_{E} f\left(s_{E}(t)\right), \\
\frac{d I(t)}{d t}=-\alpha_{I} I(t)+(1-I(t)) \beta_{I} f\left(s_{I}(t)\right) .
\end{gathered}
$$

The synaptic inputs $s_{E(t)}$ and $s_{I(t)}$ are given by Eqs. (3) and (4), respectively. We are interested in the dynamics of the deviations $V_{E(t)}$ and $V_{I(t)}$. For that, we insert Eqs. (9) in Eqs. (A1) and (A2), which leads to

$$
\begin{aligned}
\frac{d\left(E_{0}+V_{E}\right)}{d t}= & -\alpha_{E}\left(E_{0}+V_{E}\right)+\left(1-\left(E_{0}+V_{E}\right)\right) \beta_{E} f\left(s_{E}\right) \\
= & -\alpha_{E} E_{0}-\alpha_{E} V_{E}+\left(1-E_{0}\right) \beta_{E} f\left(s_{E}\right) \\
& -V_{E} \beta_{E} f\left(s_{E}\right), \\
\frac{d\left(I_{0}+V_{I}\right)}{d t}= & -\alpha_{I}\left(I_{0}+V_{I}\right)+\left(1-\left(I_{0}+V_{I}\right)\right) \beta_{I} f\left(s_{I}\right) \\
= & -\alpha_{I} I_{0}-\alpha_{I} V_{I}+\left(1-I_{0}\right) \beta_{I} f\left(s_{I}\right)-V_{I} \beta_{I} f\left(s_{I}\right) .
\end{aligned}
$$

We now replace $f\left(s_{E}\right)$ and $f\left(s_{I}\right)$ by their Taylor expansion at the second order given in Eqs. (11):

$$
\begin{aligned}
\frac{d\left(E_{0}+V_{E}\right)}{d t}= & -\alpha_{E}\left(E_{0}+V_{E}\right)+\left(1-\left(E_{0}+V_{E}\right)\right) \beta_{E} f\left(s_{E}\right) \\
= & -\alpha_{E} E_{0}-\alpha_{E} V_{E}+\left(1-E_{0}\right) \beta_{E}\left(f\left(s_{E_{0}}\right)+\delta s_{E} f^{\prime}\left(s_{E_{0}}\right)+\frac{1}{2}\left(\delta s_{E}\right)^{2} f^{\prime \prime}\left(s_{E_{0}}\right)\right) \\
& -V_{E} \beta_{E}\left(f\left(s_{E_{0}}\right)+\delta s_{E} f^{\prime}\left(s_{E_{0}}\right)+\frac{1}{2}\left(\delta s_{E}\right)^{2} f^{\prime \prime}\left(s_{E_{0}}\right)\right) \\
\frac{d\left(I_{0}+V_{I}\right)}{d t}= & -\alpha_{I}\left(I_{0}+V_{I}\right)+\left(1-\left(I_{0}+V_{I}\right)\right) \beta_{I} f\left(s_{I}\right) \\
= & -\alpha_{I} I_{0}-\alpha_{I} V_{I}+\left(1-I_{0}\right) \beta_{I}\left(f\left(s_{I_{0}}\right)+\delta s_{I} f^{\prime}\left(s_{I_{0}}\right)+\frac{1}{2}\left(\delta s_{I}\right)^{2} f^{\prime \prime}\left(s_{I_{0}}\right)\right) \\
& -V_{I} \beta_{I}\left(f\left(s_{I_{0}}\right)+\delta s_{I} f^{\prime}\left(s_{I_{0}}\right)+\frac{1}{2}\left(\delta s_{I}\right)^{2} f^{\prime \prime}\left(s_{I_{0}}\right)\right) .
\end{aligned}
$$


Taking into account the fixed point solutions Eq. (10), the equations above reduce to

$$
\begin{aligned}
& \frac{d V_{E}}{d t}=-\alpha_{E} V_{E}+\left(1-E_{0}\right) \beta_{E}\left(\delta s_{E} f^{\prime}\left(s_{E_{0}}\right)+\frac{1}{2}\left(\delta s_{E}\right)^{2} f^{\prime \prime}\left(s_{E_{0}}\right)\right)-V_{E} \beta_{E}\left(f\left(s_{E_{0}}\right)+\delta s_{E} f^{\prime}\left(s_{E_{0}}\right)+\frac{1}{2}\left(\delta s_{E}\right)^{2} f^{\prime \prime}\left(s_{E_{0}}\right)\right), \\
& \frac{d V_{I}}{d t}=-\alpha_{I} V_{I}+\left(1-I_{0}\right) \beta_{I}\left(\delta s_{I} f^{\prime}\left(s_{I_{0}}\right)+\frac{1}{2}\left(\delta s_{I}\right)^{2} f^{\prime \prime}\left(s_{I_{0}}\right)\right)-V_{I} \beta_{I}\left(f\left(s_{I_{0}}\right)+\delta s_{I} f^{\prime}\left(s_{I_{0}}\right)+\frac{1}{2}\left(\delta s_{I}\right)^{2} f^{\prime \prime}\left(s_{I_{0}}\right)\right) .
\end{aligned}
$$

We now group the terms of the equations according to different orders:

$$
\begin{aligned}
\frac{d V_{E}}{d t}= & -\alpha_{E} V_{E}+\left(1-E_{0}\right) \beta_{E} f^{\prime}\left(s_{E_{0}}\right) \delta s_{E}-\beta_{E} f\left(s_{E_{0}}\right) V_{E}+\left(1-E_{0}\right) \beta_{E}\left(\frac{1}{2}\left(\delta s_{E}\right)^{2} f^{\prime \prime}\left(s_{E_{0}}\right)\right)-\beta_{E} V_{E}\left(\delta s_{E} f^{\prime}\left(s_{E_{0}}\right)\right) \\
& -\beta_{E} V_{E}\left(\frac{1}{2}\left(\delta s_{E}\right)^{2} f^{\prime \prime}\left(s_{E_{0}}\right)\right) \\
\frac{d V_{I}}{d t}= & -\alpha_{I} V_{I}+\left(1-I_{0}\right) \beta_{I} f^{\prime}\left(s_{I_{0}}\right) \delta s_{I}-\beta_{I} f\left(s_{I_{0}}\right) V_{I}+\left(1-I_{0}\right) \beta_{I}\left(\frac{1}{2}\left(\delta s_{I}\right)^{2} f^{\prime \prime}\left(s_{I_{0}}\right)\right)-\beta_{I} V_{I}\left(\delta s_{I} f^{\prime}\left(s_{I_{0}}\right)\right) \\
& -\beta_{I} V_{I}\left(\frac{1}{2}\left(\delta s_{I}\right)^{2} f^{\prime \prime}\left(s_{I_{0}}\right)\right) .
\end{aligned}
$$

We replace $\delta s_{E}$ and $\delta s_{I}$ by their expressions:

$$
\begin{aligned}
\frac{d V_{E}}{d t}= & -\alpha_{E} V_{E}+\left(1-E_{0}\right) \beta_{E} f^{\prime}\left(s_{E_{0}}\right)\left(W_{E E} V_{E}-W_{E I} V_{I}\right)-\beta_{E} f\left(s_{E_{0}}\right) V_{E}-\underline{\beta_{E} f^{\prime}\left(s_{E_{0}}\right) V_{E}\left(W_{E E} V_{E}-W_{E I} V_{I}\right)} \\
& +\underbrace{\frac{1}{2}\left(1-E_{0}\right) \beta_{E} f^{\prime \prime}\left(s_{E_{0}}\right)\left(W_{E E}^{2} V_{E}^{2}+W_{E I}^{2} V_{I}^{2}-2 W_{E E} W_{E I} V_{E} V_{I}\right)}-\frac{1}{2} \beta_{E} f^{\prime \prime}\left(s_{E_{0}}\right) V_{E}\left(W_{E E}^{2} V_{E}^{2}+W_{E I}^{2} V_{I}^{2}-2 W_{E E} W_{E I} V_{E} V_{I}\right),
\end{aligned}
$$

$$
\begin{aligned}
\frac{d V_{I}}{d t}= & -\alpha_{I} V_{I}+\left(1-I_{0}\right) \beta_{I} f^{\prime}\left(s_{I_{0}}\right)\left(W_{I E} V_{E}-W_{I I} V_{I}\right)-\beta_{I} f\left(s_{I_{0}}\right) V_{I}-\beta_{I} f^{\prime}\left(s_{I_{0}}\right) V_{I}\left(W_{I E} V_{E}-W_{I I} V_{I}\right) \\
& +\underbrace{\frac{1}{2}\left(1-I_{0}\right) \beta_{I} f^{\prime \prime}\left(s_{I_{0}}\right)\left(W_{I E}^{2} V_{E}^{2}+W_{I I}^{2} V_{I}^{2}-2 W_{I E} W_{I I} V_{E} V_{I}\right)}-\frac{1}{2} \beta_{I} f^{\prime \prime}\left(s_{I_{0}}\right) V_{I}\left(W_{I E}^{2} V_{E}^{2}+W_{I I}^{2} V_{I}^{2}-2 W_{I E} W_{I I} V_{E} V_{I}\right) .
\end{aligned}
$$

The dynamics of the deviation from the fixed point $\left(E_{0}, I_{0}\right)$ is composed of terms of three different orders. We have terms of order 1 (linear), those of order 2 (quadratic) and finally terms of order 3 (cubic). We are particularly interested in quadratic and cubic terms, since the effect of linear terms has been studied previously. Note that quadratic terms have two origins: some come from the product of two linear terms [Eqs. (A3) and (A4), terms underline] and others from the square of the linear terms $\delta s_{E}$ and $\delta s_{I}$ [Eqs. (A3) and (A4), terms underbrace].

We are looking for a dynamics which approximates the full Wilson-Cowan model (with the sigmoid function) in the vicinity of the Hopf bifurcation. We compare the bifurcation diagrams of the approximate model Eqs. (A3) and (A4) (called the full WC3) derived above and the full Wilson-Cowan model Eqs. (A1) and (A2) (full WC). We find that the approximate model [Eqs. (A3) and (A4)] above (green curve, Fig. 14) does not describe well the full Wilson Cowan (red curve, Fig. 14) even close to the Hopf bifurcation. We discard quadratic terms coming from the square of the terms $\delta s_{E}$ and $\delta s_{I}$. The new dynamics [Eqs. (A5) and (A6), called the WC3] without these terms (blue curve, Fig. 14) now approximates well the full Wilson Cowan (red curve, Fig. 14) in the vicinity of the Hopf bifurcation. In this work, we therefore use these new dynamics Eqs. (A5) and (A6) since they are a better approximation of the Wilson-Cowan for the parameter regimes of interest. To obtain its stochastic version named the SWC3 to which we eventually apply the SAM, we add the noise terms $\eta_{E}$ and $\eta_{I}$. The resulting dynamics are given by Eqs. (13) and (14). We note that the discarded quadratic terms would not have contributed to the SAM approximation that we compute.

$$
\begin{aligned}
\frac{d V_{E}}{d t}= & -\alpha_{E} V_{E}+\left(1-E_{0}\right) \beta_{E} f^{\prime}\left(s_{E_{0}}\right)\left(W_{E E} V_{E}-W_{E I} V_{I}\right)-\beta_{E} f\left(s_{E_{0}}\right) V_{E}-\underline{\beta_{E} f^{\prime}\left(s_{E_{0}}\right) V_{E}\left(W_{E E} V_{E}-W_{E I} V_{I}\right)} \\
& -\frac{1}{2} \beta_{E} f^{\prime \prime}\left(s_{E_{0}}\right) V_{E}\left(W_{E E}^{2} V_{E}^{2}+W_{E I}^{2} V_{I}^{2}-2 W_{E E} W_{E I} V_{E} V_{I}\right) \\
\frac{d V_{I}}{d t}= & -\alpha_{I} V_{I}+\left(1-I_{0}\right) \beta_{I} f^{\prime}\left(s_{I_{0}}\right)\left(W_{I E} V_{E}-W_{I I} V_{I}\right)-\beta_{I} f\left(s_{I_{0}}\right) V_{I}-\underline{\beta_{I} f^{\prime}\left(s_{I_{0}}\right) V_{I}\left(W_{I E} V_{E}-W_{I I} V_{I}\right)} \\
& -\frac{1}{2} \beta_{I} f^{\prime \prime}\left(s_{I_{0}}\right) V_{I}\left(W_{I E}^{2} V_{E}^{2}+W_{I I}^{2} V_{I}^{2}-2 W_{I E} W_{I I} V_{E} V_{I}\right) .
\end{aligned}
$$




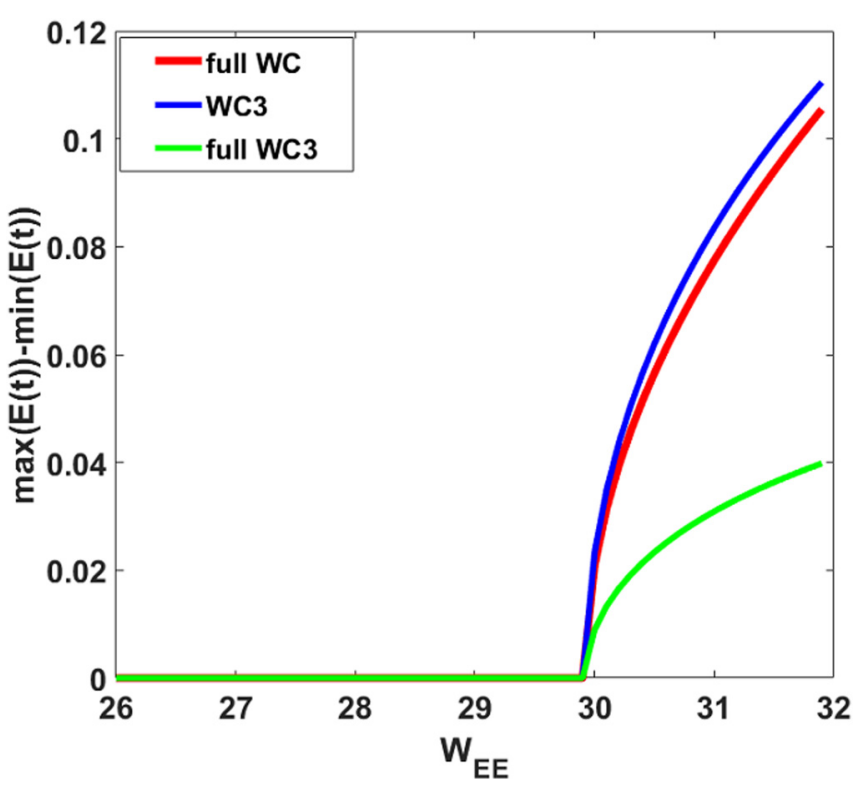

FIG. 14. Bifurcation diagram of the full Wilson-Cowan model and its approximate versions obtained after a Taylor expansion of the sigmoid function. The red curve represents the full (with the sigmoid function) deterministic Wilson Cowan (WC) dynamics [Eqs. (A1) and (A2)]. The green curve is the bifurcation diagram of the approximate Wilson Cowan with the Taylor expansion of the sigmoid function at order 2 (full WC3), Eqs. (A3) and (A4). Finally, the blue curve is obtained from the full WC3 after discarding the quadratic terms coming from the square of the quantities $\delta s_{E}$ and $\delta s_{I}$ [see Eqs. (A5) and (A6)]. All the curves were obtained using a fourth order Runge-Kutta iterative scheme with time step $d t=0.025 \mathrm{~ms}$.

\section{Expansion of the stochastic averaging method (SAM)}

The drift two-dimensional function $m=\left(\begin{array}{c}m_{1}(t) \\ m_{2}(t)\end{array}\right)$ obtained from the stochastic averaging method (SAM) can be expanded as follows:

$$
\begin{aligned}
m_{1}(t)= & T^{a v}\left(E\left\{F_{1}(t)\right\}+\int_{-\infty}^{0} E\left\{\frac{\partial G_{1}(t)}{\partial Z_{E}} G_{1}(t+\tau)\right\} d \tau\right. \\
& \left.+\int_{-\infty}^{0} E\left\{\frac{\partial G_{1}(t)}{\partial \phi_{E}} G_{2}(t+\tau)\right\} d \tau\right), \\
m_{2}(t)= & T^{a v}\left(E\left\{F_{2}(t)\right\}+\int_{-\infty}^{0} E\left\{\frac{\partial G_{2}(t)}{\partial Z_{E}} G_{1}(t+\tau)\right\} d \tau\right. \\
& \left.+\int_{-\infty}^{0} E\left\{\frac{\partial G_{2}(t)}{\partial \phi_{E}} G_{2}(t+\tau)\right\} d \tau\right) .
\end{aligned}
$$

The coefficients $h_{i j}$ of the diffusion matrix $h$ can also be obtained as

$$
h_{11}^{2}+h_{12}^{2}=T^{a v}\left(\int_{-\infty}^{\infty} E\left\{G_{1}(t) G_{1}(t+\tau)\right\} d \tau\right),
$$

$$
\begin{aligned}
h_{11} h_{21}+h_{12} h_{22} & =T^{a v}\left(\int_{-\infty}^{\infty} E\left\{G_{1}(t) G_{2}(t+\tau)\right\} d \tau\right), \\
h_{11} h_{21}+h_{12} h_{22} & =T^{a v}\left(\int_{-\infty}^{\infty} E\left\{G_{2}(t) G_{1}(t+\tau)\right\} d \tau\right), \\
h_{21}^{2}+h_{22}^{2} & =T^{a v}\left(\int_{-\infty}^{\infty} E\left\{G_{2}(t) G_{2}(t+\tau)\right\} d \tau\right) .
\end{aligned}
$$

When the noises acting on the two variables are uncorrelated and only additive, an easy choice of the non diagonal components of the matrix $h$ can be made $\left(h_{12}=h_{21}=0\right)$. This leads to

$$
\begin{aligned}
& h_{11}^{2}=T^{a v}\left(\int_{-\infty}^{\infty} E\left\{G_{1}(t) G_{1}(t+\tau)\right\} d \tau\right), \\
& h_{12}=0, \\
& h_{21}=0, \\
& h_{22}^{2}=T^{a v}\left(\int_{-\infty}^{\infty} E\left\{G_{2}(t) G_{2}(t+\tau)\right\} d \tau\right) .
\end{aligned}
$$

If instead the noises are correlated or multiplicative, such an easy choice could not be made. This will lead to the following system:

$$
\begin{aligned}
& d Z_{E}(t)=m_{1}(t) d t+h_{11} d W_{1}(t)+h_{12} d W_{2}(t), \\
& d \phi_{E}(t)=m_{2}(t) d t+h_{12} d W_{1}(t)+h_{22} d W_{2}(t) .
\end{aligned}
$$

This system of coupled equations can be approximated as follows:

$$
\begin{aligned}
& d Z_{E}(t)=m_{1}(t) d t+\sqrt{h_{11}^{2}+h_{12}^{2}} d W_{3}(t), \\
& d \phi_{E}(t)=m_{2}(t) d t+\sqrt{h_{12}^{2}+h_{22}^{2}} d W_{4}(t) .
\end{aligned}
$$

The noises terms $h_{11} d W_{1}(t)+h_{12} d W_{2}(t)$ and $h_{12} d W_{1}(t)+$ $h_{22} d W_{2}(t)$ are replaced by $\sqrt{h_{11}^{2}+h_{12}^{2}} d W_{3}(t)$ and $\sqrt{h_{12}^{2}+h_{22}^{2}} d W_{4}(t)$ respectively, where $d W_{3}(t)$ and $d W_{4}(t)$ are two independent Wiener processes. The new noise coefficients depend only on the diagonal elements of the matrix $G_{t} G_{t+\tau}^{\prime}$. This avoids solving the nonlinear four-dimensional equations in $h_{i j}$ and allows an easy derivation of the envelope and phase dynamics.

The functions $F=\left(\begin{array}{c}F_{1}(t) \\ F_{2}(t)\end{array}\right)$ and $G=\left(\begin{array}{l}G_{1}(t) \\ G_{2}(t)\end{array}\right)$ are defined from Eqs. (21) and (22). They are replaced by $F^{k}$ and $G^{k}$ defined from Eqs. (55) and (56) for the case of networks of oscillators. We recall that $T^{a v}$ is the time averaging operator defined as

$$
T^{a v}(.)=\frac{1}{T_{0}} \int_{t_{0}}^{t_{0}+T_{0}}(.) d t,
$$

and $E$. denotes the expectation operator (i.e., average over realizations).
[1] B. Lindner, K. Dierkes, and F. Jülicher, Local Exponents of Nonlinear Compression in Periodically Driven Noisy Oscillators, Phys. Rev. Lett. 103, 250601 (2009).

[2] E. Lowet, M. Roberts, A. Hadjipapas, A. Peter, J. van der Eerden, and P. De Weerd, Input-dependent frequency modulation of cortical gamma oscillations shapes spatial synchronization and enables phase coding, PLoS Comput. Biol. 11, e1004072 (2015).

[3] E. Lowet, M. J. Roberts, A. Peter, B. Gips, and P. De Weerd, A quantitative theory of gamma 
synchronization in macaque v1, Elife 6, e26642 (2017).

[4] R. M. May, Limit cycles in predator-prey communities, Science 177, 900 (1972).

[5] A. Hastings, K. C. Abbott, K. Cuddington, T. Francis, G. Gellner, Y.-C. Lai, A. Morozov, S. Petrovskii, K. Scranton, and M. L. Zeeman, Transient phenomena in ecology, Science 361, eaat6412 (2018).

[6] H. Kresnel, Semiconductor Lasers and Heterojunction LEDs (Elsevier, Amsterdam, 2012).

[7] A. Goldbeter, Biochemical Oscillations and Cellular Rhythms: The Molecular Bases of Periodic and Chaotic Behavior (Cambridge University Press, Cambridge, England, 1997).

[8] A. Goldbeter, Modelling biochemical oscillations and cellular rhythms, Curr. Sci. 73, 933 (1997).

[9] A. Goldbeter, Computational approaches to cellular rhythms, Nature (London) 420, 238 (2002).

[10] A. J. McKane, J. D. Nagy, T. J. Newman, and M. O. Stefanini, Amplified biochemical oscillations in cellular systems, J. Stat. Phys. 128, 165 (2007).

[11] B. Novák and J. J. Tyson, Design principles of biochemical oscillators, Nat. Rev. Mol. Cell Biol. 9, 981 (2008).

[12] N. Barkai and S. Leibler, Circadian clocks limited by noise, Nature (London) 403, 267 (2000).

[13] D. Bell-Pedersen, V. M. Cassone, D. J. Earnest, S. S. Golden, P. E. Hardin, T. L. Thomas, and M. J. Zoran, Circadian rhythms from multiple oscillators: lessons from diverse organisms, Nat. Rev. Genet. 6, 544 (2005).

[14] T. Danino, O. Mondragón-Palomino, L. Tsimring, and J. Hasty, A synchronized quorum of genetic clocks, Nature (London) 463, 326 (2010).

[15] L. S. Tsimring, Noise in biology, Rep. Prog. Phys. 77, 026601 (2014).

[16] J. Stricker, S. Cookson, M. R. Bennett, W. H. Mather, L. S. Tsimring, and J. Hasty, A fast, robust and tunable synthetic gene oscillator, Nature (London) 456, 516 (2008).

[17] M. O. Din, T. Danino, A. Prindle, M. Skalak, J. Selimkhanov, K. Allen, E. Julio, E. Atolia, L. S. Tsimring, S. N. Bhatia et al., Synchronized cycles of bacterial lysis for in vivo delivery, Nature (London) 536, 81 (2016).

[18] O. Mondragón-Palomino, T. Danino, J. Selimkhanov, L. Tsimring, and J. Hasty, Entrainment of a population of synthetic genetic oscillators, Science 333, 1315 (2011).

[19] L. Glass, Synchronization and rhythmic processes in physiology, Nature (London) 410, 277 (2001).

[20] M. R. Guevara, L. Glass, and A. Shrier, Phase locking, period-doubling bifurcations, and irregular dynamics in periodically stimulated cardiac cells, Science 214, 1350 (1981).

[21] R. E. Dolmetsch, K. Xu, and R. S. Lewis, Calcium oscillations increase the efficiency and specificity of gene expression, Nature (London) 392, 933 (1998).

[22] J. Sneyd, J. Keizer, and M. J. Sanderson, Mechanisms of calcium oscillations and waves: a quantitative analysis, FASEB J. 9, 1463 (1995)

[23] J. P. Aparicio and H. G. Solari, Sustained oscillations in stochastic systems, Math. Biosci. 169, 15 (2001).

[24] G. Rozhnova and A. Nunes, Fluctuations and oscillations in a simple epidemic model, Phys. Rev. E 79, 041922 (2009).
[25] D. Mollison, Simplifying simple epidemic models, Nature (London) 310, 224 (1984).

[26] A. J. McKane and T. J. Newman, Predator-Prey Cycles from Resonant Amplification of Demographic Stochasticity, Phys. Rev. Lett. 94, 218102 (2005).

[27] G. Buzsaki, Rhythms of Brain (Oxford University Press, Oxford, England, 2006).

[28] G. Buzsáki and X.-J. Wang, Mechanisms of gamma oscillations, Annu. Rev. Neurosci. 35, 203 (2012).

[29] M. Bartos, I. Vida, and P. Jonas, Synaptic mechanisms of synchronized gamma oscillations in inhibitory interneuron networks, Nat. Rev. Neurosci. 8, 45 (2007).

[30] X. Jia and A. Kohn, Gamma rhythms in the brain, PLoS Biol. 9, e1001045 (2011).

[31] E. Wallace, M. Benayoun, W. Van Drongelen, and J. D. Cowan, Emergent oscillations in networks of stochastic spiking neurons, PLoS One 6, e14804 (2011).

[32] J. D. Salvi, D. Ó. Maoiléidigh, and A. J. Hudspeth, Identification of bifurcations from observations of noisy biological oscillators, Biophys. J. 111, 798 (2016).

[33] V. M. Eguíluz, M. Ospeck, Y. Choe, A. J. Hudspeth, and M. O. Magnasco, Essential Nonlinearities in Hearing, Phys. Rev. Lett. 84, 5232 (2000).

[34] S. Palva and J. M. Palva, New vistas for $\alpha$-frequency band oscillations, Trends Neurosci. 30, 150 (2007).

[35] F. H. L. Da Silva, J. P. Pijn, D. Velis, and P. C. G. Nijssen, Alpha rhythms: noise, dynamics and models, Int. J. Psychophysiol. 26, 237 (1997).

[36] A. K. Engel and P. Fries, Beta-band oscillations - signalling the status quo? Curr. Opin. Neurobiol. 20, 156 (2010).

[37] N. Kopell, G. B. Ermentrout, M. A. Whittington, and R. D. Traub, Gamma rhythms and beta rhythms have different synchronization properties, Proc. Natl. Acad. Sci. USA 97, 1867 (2000)

[38] K.-H. Lee, L. M. Williams, M. Breakspear, and E. Gordon, Synchronous gamma activity: a review and contribution to an integrative neuroscience model of schizophrenia, Brain Res. Rev. 41, 57 (2003).

[39] C. A. Bosman, J.-M. Schoffelen, N. Brunet, R. Oostenveld, A. M. Bastos, T. Womelsdorf, B. Rubehn, T. Stieglitz, P. De Weerd, and P. Fries, Attentional stimulus selection through selective synchronization between monkey visual areas, Neuron 75, 875 (2012).

[40] P. Fries, A mechanism for cognitive dynamics: neuronal communication through neuronal coherence, Trends Cognit. Sci. 9, 474 (2005).

[41] J. Yamamoto, J. Suh, D. Takeuchi, and S. Tonegawa, Successful execution of working memory linked to synchronized high-frequency gamma oscillations, Cell 157, 845 (2014).

[42] M. Lundqvist, J. Rose, P. Herman, S. L. Brincat, T. J. Buschman, and E. K. Miller, Gamma and beta bursts underlie working memory, Neuron 90, 152 (2016).

[43] P. Fries, Rhythms for cognition: communication through coherence, Neuron 88, 220 (2015).

[44] A. Palmigiano, T. Geisel, F. Wolf, and D. Battaglia, Flexible information routing by transient synchrony, Nat. Neurosci. 20, 1014 (2017).

[45] P. Brown, Abnormal oscillatory synchronisation in the motor system leads to impaired movement, Curr. Opin. Neurobiol. 17, 656 (2007). 
[46] C. Hammond, H. Bergman, and P. Brown, Pathological synchronization in Parkinson's disease: Networks, models and treatments, Trends Neurosci. 30, 357 (2007).

[47] C. S. Herrmann and T. Demiralp, Human EEG gamma oscillations in neuropsychiatric disorders, Clin. Neurophysiol. 116, 2719 (2005).

[48] K. C. A. Wedgwood, K. K. Lin, R. Thul, and S. Coombes, Phase-amplitude descriptions of neural oscillator models, J. Math. Neurosci. 3, 2 (2013).

[49] P. Ashwin, S. Coombes, and R. Nicks, Mathematical frameworks for oscillatory network dynamics in neuroscience, J. Math. Neurosci. 6, 2 (2016).

[50] S. Shirasaka, W. Kurebayashi, and H. Nakao, Phase-amplitude reduction of transient dynamics far from attractors for limitcycling systems, Chaos: An Interdisciplinary Journal of Nonlinear Science 27, 023119 (2017).

[51] A. Mauroy and I. Mezić, Global computation of phaseamplitude reduction for limit-cycle dynamics, Chaos $\mathbf{2 8}$, 073108 (2018)

[52] M. Bonnin, Amplitude and phase dynamics of noisy oscillators, Int. J. Circuit Theory Appl. 45, 636 (2017).

[53] M. Bonnin, F. L. Traversa, and F. Bonani, Colored noise in oscillators. phase-amplitude analysis and a method to avoid the ito-stratonovich dilemma, IEEE Trans. Circuits Syst. I: Regular Papers 66, 3917 (2019).

[54] M. Bonnin, F. Bonani, and F. L. Traversa, Kuramoto-like model of noisy oscillators, in 2017 European Conference on Circuit Theory and Design (ECCTD) (IEEE, 2017), pp. 1-4.

[55] W. Ebeling, H. Herzel, and E. E. Selkov, The influence of noise on an oscillating glycolytic model, Studia Biophysica 98, 147 (1983).

[56] L. Fronzoni, R. Mannella, P. V. E. McClintock, and F. Moss, Postponement of Hopf bifurcations by multiplicative colored noise, Phys. Rev. A 36, 834 (1987).

[57] S. Kabashima and T. Kawakubo, Observation of a noiseinduced phase transition in a parametric oscillator, Phys. Lett. A 70, 375 (1979).

[58] P. C. Bressloff and J. N. MacLaurin, Phase reduction of stochastic biochemical oscillators, SIAM J. Appl. Dynamical Systems 19, 151 (2020).

[59] P. E. Greenwood, M. D. McDonnell, and L. M. Ward, Dynamics of gamma bursts in local field potentials, Neural Comput. 27, 74 (2015).

[60] A. S. Powanwe and A. Longtin, Determinants of brain rhythm burst statistics, Sci. Rep. 9, 18335 (2019).

[61] D. Xing, Y. Shen, S. Burns, C.-I. Yeh, R. Shapley, and W. $\mathrm{Li}$, Stochastic generation of gamma-band activity in primary visual cortex of awake and anesthetized monkeys, J. Neurosci. 32, 13873 (2012).

[62] D. Nikolić, P. Fries, and W. Singer, Gamma oscillations: precise temporal coordination without a metronome, Trends Cognit. Sci. 17, 54 (2013).

[63] S. P. Burns, D. Xing, and R. M. Shapley, Is gamma-band activity in the local field potential of $\mathrm{v} 1$ cortex a clock or filtered noise? J. Neurosci. 31, 9658 (2011).

[64] P. H. Coullet, C. Elphick, and E. Tirapegui, Normal form of a hopf bifurcation with noise, Phys. Lett. A 111, 277 (1985).

[65] R. Lefever and J. W. Turner, Sensitivity of a Hopf Bifurcation to Multiplicative Colored Noise, Phys. Rev. Lett. 56, 1631 (1986).
[66] Na Yu, R. Kuske, and Y. X. Li, Stochastic phase dynamics: Multiscale behavior and coherence measures, Phys. Rev. E 73, 056205 (2006).

[67] A. S. Powanwe and A. Longtin, Brain rhythm bursts are enhanced by multiplicative noise, Chaos 31, 013117 (2021).

[68] P. E. Greenwood, M. D. McDonnell, and L. M. Ward, A Kuramoto coupling of quasi-cycle oscillators with application to neural networks, J. Coupled Systems Multiscale Dynamics 4, 1 (2016).

[69] A. S. Powanwe and A. Longtin, Phase dynamics of delaycoupled quasi-cycles with application to brain rhythms, Phys. Rev. Res. 2, 043067 (2020).

[70] R. L. Stratonovich, Topics in the Theory of Random Noise (CRC, 1967), Vol. 2.

[71] R. Z. Khas minskii, A limit theorem for the solutions of differential equations with random right-hand sides, Theory Probab. Appl. 11, 390 (1966).

[72] J. B. Roberts and P. D. Spanos, Stochastic averaging: an approximate method of solving random vibration problems, Int. J. Non Linear Mech. 21, 111 (1986).

[73] H. R. Wilson and J. D. Cowan, Excitatory and inhibitory interactions in localized populations of model neurons, Biophys. J. 12, 1 (1972)

[74] Y. Cao, H. Wang, Q. Ouyang, and Y. Tu, The free-energy cost of accurate biochemical oscillations, Nat. Phys. 11, 772 (2015).

[75] G. Deco, J. Cabral, M. W. Woolrich, A. B. A. Stevner, T. J. Van Hartevelt, and M. L. Kringelbach, Single or multiple frequency generators in on-going brain activity: A mechanistic whole-brain model of empirical meg data, Neuroimage 152, 538 (2017).

[76] G. Deco, M. L. Kringelbach, V. K. Jirsa, and P. Ritter, The dynamics of resting fluctuations in the brain: metastability and its dynamical cortical core, Sci. Rep. 7, 3095 (2017).

[77] B. M. Jobst, R. Hindriks, H. Laufs, E. Tagliazucchi, G. Hahn, A. Ponce-Alvarez, A. B. A. Stevner, M. L. Kringelbach, and G. Deco, Increased stability and breakdown of brain effective connectivity during slow-wave sleep: mechanistic insights from whole-brain computational modelling, Sci. Rep. 7, 4634 (2017).

[78] S. H. Strogatz, Nonlinear Dynamics and Chaos: with Application to Physics, Biology, Chemistry and Engineering (Perseus Books, Reading, Massachusetts, 1994), Vol. 1.

[79] C. W. Gardiner et al., Handbook of Stochastic Methods (Springer Berlin, Berlin, 1985), Vol. 3.

[80] A. Daffertshofer and B. van Wijk, On the influence of amplitude on the connectivity between phases, Frontiers Neuroinformatics 5, 6 (2011).

[81] H. G. Schuster and P. Wagner, A model for neuronal oscillations in the visual cortex, Biol. Cybern. 64, 77 (1990).

[82] S. T. Ariaratnam and T. K. Srikantaiah, Parametric instabilities in elastic structures under stochastic loading, J. Structural Mech. 6, 349 (1978).

[83] B. Pietras, Modeling phase synchronization of interacting neural populations, Ph.D. thesis, VRIJE Universiteit, AMSTERDAM, the Netherlands, Lancaster University, Lancaster, United Kingdom, 2018.

[84] A. Hyafil, A.-L. Giraud, L. Fontolan, and B. Gutkin, Neural cross-frequency coupling: connecting architectures, 
mechanisms, and functions, Trends Neurosci. 38, 725 (2015).

[85] R. E. Mirollo and S. H. Strogatz, Amplitude death in an array of limit-cycle oscillators, J. Stat. Phys. 60, 245 (1990).

[86] P. C. Matthews and S. H. Strogatz, Phase Diagram for the Collective Behavior of Limit-Cycle Oscillators, Phys. Rev. Lett. 65, 1701 (1990).

[87] H. G. Schuster and P. Wagner, Mutual entrainment of two limit cycle oscillators with time delayed coupling, Prog. Theor. Phys. 81, 939 (1989).

[88] M. K. Stephen Yeung and S. H. Strogatz, Time Delay in the Kuramoto Model of Coupled Oscillators, Phys. Rev. Lett. 82, 648 (1999).

[89] E. Niebur, H. G. Schuster, and D. M. Kammen, Collective Frequencies and Metastability in Networks of Limit-Cycle Oscillators with Time Delay, Phys. Rev. Lett. 67, 2753 (1991).

[90] Y. Nakamura, F. Tominaga, and T. Munakata, Clustering behavior of time-delayed nearest-neighbor coupled oscillators, Phys. Rev. E 49, 4849 (1994).

[91] I. V. Tyulkina, D. S. Goldobin, L. S. Klimenko, and A. Pikovsky, Dynamics of Noisy Oscillator Populations Beyond the Ott-Antonsen Ansatz, Phys. Rev. Lett. 120, 264101 (2018).

[92] J. Cabral, E. Hugues, O. Sporns, and G. Deco, Role of local network oscillations in resting-state functional connectivity, Neuroimage 57, 130 (2011).

[93] Y. Kuramoto, Chemical Oscillations, Waves, and Turbulence (Courier Corporation, 2003).

[94] J. A. Acebrón, L. L. Bonilla, C. J. P. Vicente, F. Ritort, and R. Spigler, The kuramoto model: A simple paradigm for synchronization phenomena, Rev. Mod. Phys. 77, 137 (2005).
[95] M. Komarov and A. Pikovsky, Multiplicity of Singular Synchronous States in the Kuramoto Model of Coupled Oscillators, Phys. Rev. Lett. 111, 204101 (2013).

[96] F. Peter and A. Pikovsky, Transition to collective oscillations in finite Kuramoto ensembles, Phys. Rev. E 97, 032310 (2018).

[97] E. Montbrió and D. Pazó, Kuramoto Model for ExcitationInhibition-Based Oscillations, Phys. Rev. Lett. 120, 244101 (2018).

[98] H. Hong and S. H. Strogatz, Kuramoto Model of Coupled Oscillators with Positive and Negative Coupling Parameters: an Example of Conformist and Contrarian Oscillators, Phys. Rev. Lett. 106, 054102 (2011).

[99] C. Kirst, M. Timme, and D. Battaglia, Dynamic information routing in complex networks, Nat. Commun. 7, 11061 (2016).

[100] A. Pikovsky, J. Kurths, M. Rosenblum, and J. Kurths, Synchronization: A Universal Concept in Nonlinear Sciences (Cambridge University Press, Cambridge, England, 2003), Vol. 12.

[101] F. Varela, J.-P. Lachaux, E. Rodriguez, and J. Martinerie, The brainweb: phase synchronization and large-scale integration, Nat. Rev. Neurosci. 2, 229 (2001).

[102] D. M. Abrams, R. Mirollo, S. H. Strogatz, and D. A. Wiley, Solvable Model for Chimera States of Coupled Oscillators, Phys. Rev. Lett. 101, 084103 (2008).

[103] A. Yeldesbay, A. Pikovsky, and M. Rosenblum, Chimeralike States in an Ensemble of Globally Coupled Oscillators, Phys. Rev. Lett. 112, 144103 (2014).

[104] Á. Byrne, R. D. ODea, M. Forrester, J. Ross, and S. Coombes, Next-generation neural mass and field modeling, J. Neurophysiol. 123, 726 (2020). 